\title{
Modelling and Analysis of Virotherapy of Cancer Using an Efficient Hybrid Soft Computing Procedure
}

\author{
M. Fawad Khan $\mathbb{D}^{1},{ }^{1}$ Ebenezer Bonyah $\left(\mathbb{D},{ }^{2}\right.$ Fahad Sameer Alshammari $\left(\mathbb{D},{ }^{3}\right.$ \\ Syed Muhammad Ghufran, ${ }^{1}$ and Muhammad Sulaiman (iD) ${ }^{1}$ \\ ${ }^{1}$ Department of Mathematics, Abdul Wali Khan University, Mardan 23200, KP, Pakistan \\ ${ }^{2}$ Department of Mathematics Education, \\ Akenten Appiah-Menka University of Skills Training and Entrepreneurial Development, Ghana \\ ${ }^{3}$ Department of Mathematics, College of Science and Humanities in Alkharj, Prince Sattam bin Abdulaziz University, \\ Al-Kharj 11942, Saudi Arabia
}

Correspondence should be addressed to Ebenezer Bonyah; ebbonya@gmail.com

Received 23 August 2021; Accepted 8 December 2021; Published 7 January 2022

Academic Editor: Sampath Pradeep

Copyright (c) 2022 M. Fawad Khan et al. This is an open access article distributed under the Creative Commons Attribution License, which permits unrestricted use, distribution, and reproduction in any medium, provided the original work is properly cited.

\section{Introduction}

The therapy for cancer is determined on the stage of the disease. The TNM technique is used to describe the stage of cancer depending on the growth of tumor $(T)$ and indicates spreading to lymph nodes $(N)$ or if it has metastasized $(M)$ to other sites of the body. Generally, cancer is discussed in five stages. Stage 0 and 1 are considered as initial stages for tumor and are in the place where it starts. Stages 2 and 3 indicate spreading to the nearby cells/tissues and lymph nodes. Stage 4 indicates the spreading of cancer has been started and affects nearby tissues. As per the stage of cancer, different treatments are suggested to the patients, such as immunotherapy, immunotherapy, and chemotherapy. In some cases, more than one treatment is advised as a combination to shrink the size of tumor and for the removal of the tumor. In this series of treatments, virotherapy is considered as a promising therapy for cancer. Development in genetic research, mainly in genetic engineering which involves modification of viruses genetically for enhancement of the oncolytic efficacy and selectivity, has advanced and accelerated the research. These viruses hit the tumor and replicate the harmful cells without affecting the healthy cells. Measles and chickenpox are reported as useful viruses for leukemia treatment $[1,2]$. For understanding the interaction between viruses and tumor cells, developing clinically predictive virotherapy models is difficult [3]. Nevertheless, this model presents precious biological insight. For improving the outcome of this therapy, there are a variety of treatment options that might be proposed. The dynamic behavior of virotherapy is described by many mathematical models. Some of them only describe the interaction between virus-infected and uninfected tumor cells $[4,5]$. Meanwhile, many discus the dynamics through immune response toward viruses $[6,7]$. Moreover, there are some models which work on free viruses without taking immune response into a reaction of considering viruses [8-12]. Many models dealt with immune reaction as a separate model [13]. Moreover, the growth of tumor with undamped oscillatory cancer cells is used to transient diminution of the severity of disease. Wodarz et al. found that oscillatory behavior occurs in model simulations $[4,8]$ and in vivo tests, and it is also observed clinically $[10,14]$. Despite all these promising results found through clinical studies, the antitumor intended results (treatment) of viral therapy alone are limited and placed in stage 3 or higher. The efficacy can be improved by a combination of therapies for stage 3 or later stages. Synergistic therapeutic effects are found through experimental data as demonstrated by Freytag et al. [15] and Touchefeu et al. [16] or chemotherapy by You et al. [17]. 
Furthermore, for numerical experimentation, the model by Dingli et al. [18] and Tao [19] evaluated a combination of two effects, that is, radiotherapy and virotherapy. Dingli et al. [18] proposed a model for treatment in the first phase for the dynamic of tumor undergoing virotherapy. Then, later on, the extension is made to the model for the second phase treatment. The models are driven by first-order ordinary differential equations (ODEs) and are based on population dynamics. The qualitative evaluation revealed the existence of equilibrium point and success ratio that is fully or partially successful.

In this recent work, a model presented is considered as a special case of Dingli et al.'s model [18]; in this, assumption is made on logistic growth of a tumor, and this model discussed cancer that is aggressive in nature. Thus, the replication of tumors is accordingly to their size. So, cancer spreads fast and is considered as stage 3 cancer, in a very short period. It is considered an approximation that is reasonable for growth of the tumors under the influence of virotherapy, since the time of interaction is very short [12]. Moreover, this model is different from Dingli et al.'s model [18]. According to Bajzer et al. and Tian et al. [8, 9], if a virus infects a cell, then it is capable of infecting other cells. As a result, the mathematical term $(-\beta I V)$ describes the elimination of viruses included in free viruses $(V)$ due to infection of uninfected cells $(I)$. In this work, the virotherapy is evaluated for the investigation of possible ways for successful treatment of cancer.

The solutions of mathematical models grab the attention of researchers and were solved by many local and global search techniques. Many nature-inspired and hybrid methods were introduced. Solution through soft computing is much effective and interesting. Few of them include the corneal model for eye surgery solved by fractional-order DPSO algorithm [20], model for the oscillatory behavior of the heart solved by the neuroevolutionary approach [21], temperature profiles in longitudinal fin designs by the neuroevolutionary approach [22], hybrid metaheuristic based on neurocomputing [23], dust density model solved through finite difference-based numerical computing [24], flow with stream-wise pressure gradient [25], influenza disease modelling through soft computing [26], nonlinear SITR model for novel COVID-19 dynamics [27], SIR nonlinear model based on dengue fever [28], magnetic dipole, higher-order chemical process for steady micropolar fluid, NAR-RBFs neural network for a nonlinear dusty plasma system [29], tumour virotherapy model with standard incident rate [11], NIS reporter gene for optimizing oncolytic virotherapy [30], PV-wind-fuel cell system [31], coronavirus disease (COVID-19) containing asymptomatic and symptomatic classes [32], discovery in the diagnosis of coronary artery disease [33], fractional-order modified SEIR model [34], and rainfall-dependent model for the seasonal Aedes [35]. In a similar way, we present an artificial neural network-based hybridization of Sine-Cosine Algorithm (SCA) and the Sequential Quadratic Programming (SQP) technique for solving cancer virotherapy. The dynamics of the cancer virotherapy model is evaluated as follows: (i) Variation in stages of cancer, i.e., initial condition of the model

(ii) Variation in the burst size parameter's inverse $g$

(iii) Variation in $f$, i.e., the ratio between tumor growth $(r)$ and rate of cell lysis $(\delta)$

(iv) Variation in $h$, i.e., the ratio between viral decay $(\alpha)$ and rate of cell lysis $(\delta)$

The rest of the paper is arranged as follows: Section 2 presents the model description and its stability, Section 3 presents the proposed methodology for the solution of the CVRT model, Section 4 consists of discussion and results, and five problems are given based on different values of parameter and initial conditions, and Section 5 concludes the work.

\section{Model Description}

For the treatment of cancer, this mathematical model describes the interactions between cancer and virus. The model is described by a nonlinear ODE system given in (1); all of the variables and parameters in this system are nonnegative. In the model, the population is described in the following three variables:

(i) $I(t)$ : the population of uninfected cells

(ii) $U(t)$ : the population of infected cells

(iii) $V(t)$ : the population of free viruses

$t$ represents time. The tumor is assumed as aggressive, which means that the replication of cells is proportional to its size. That is why exponential growth is considered as a growth rate $r$. Furthermore, when the free cells start encountering the uninfected cell, the cells infect with rate $\beta$. Moreover, the population of viruses decreases accordingly. The replication of virus is performed within infected cells, spreading to its lysis with rate $\delta$. In response to this, fresh virion particles are being created having burst size b. All particles of free virus should be removed from the body with rate $\alpha$. Details of parameters are given in Table 1.

For modelling cancer virotherapy, mass action law is utilized. We consider the proportionality of rate of infection of uninfected cells to the population of virus [36]. Many such models were presented which have resemblance with this model $[8,37,38]$. The mathematical model is described with the help of ODEs as follows:

$$
\begin{aligned}
& \frac{\mathrm{d} I}{\mathrm{~d} t}=r I-\beta I V, \\
& \frac{\mathrm{d} U}{\mathrm{~d} t}=\beta I V-\delta U, \\
& \frac{\mathrm{d} U}{\mathrm{~d} t}=b \delta U-\beta I V-\alpha V,
\end{aligned}
$$

with the following initial conditions: 
TABle 1: Parameters and values for system (1) taken from [10, 41].

\begin{tabular}{lccc}
\hline Parameter & Description & Value & Units \\
\hline$r$ & Tumor growth rate & $2 \times 10^{-2}$ & $1 / \mathrm{h}$ \\
$\beta$ & Infection rate of the virus & $7 / 10 \times 10^{-9}$ & $\mathrm{~mm}{ }^{3} / \mathrm{h}$ virus \\
$\delta$ & Death rate of infected tumor cells & $1 / 18$ & $1 / \mathrm{h}$ \\
$b$ & Burst size of free virus & 50 & Viruses $/$ cell \\
$\alpha$ & Clearance rate of the virus & 0.00842 & $1 / \mathrm{h}$ \\
\hline
\end{tabular}

$$
\begin{gathered}
I(0)=I_{0} \geq 0, \\
U(0)=U_{0} \geq 0, \\
V(0)=V_{0} \geq 0 .
\end{gathered}
$$

To reduce the number of the parameters in the model, system (1) is nondimensionalized by setting

$$
\begin{gathered}
t=\frac{1}{\delta} \tau, \\
I=\frac{\delta}{b \beta} \bar{I}, \\
U=\frac{\delta}{b \beta} \bar{U}, \\
V=\frac{\delta}{\beta} \bar{V},
\end{gathered}
$$

$$
\text { and letting } \begin{aligned}
f & =\frac{r}{\delta}, \\
h & =\frac{\alpha}{\delta}, \\
g & =\frac{1}{b} .
\end{aligned}
$$

To make things easier, we will remove all of the bars and write $\tau$ as $t$; thus, system (1) takes the form

$$
\begin{array}{r}
\frac{\mathrm{d} I}{\mathrm{~d} t}-f I+I v=0 \\
\frac{\mathrm{d} U}{\mathrm{~d} t}-I V+U=0 \\
\frac{\mathrm{d} V}{\mathrm{~d} t}-U+g I V+h V=0
\end{array}
$$

with the following initial conditions:

$$
\begin{aligned}
I(0) & =\frac{b \beta I_{0}}{\delta}, \\
U(0) & =\frac{b \beta U_{0}}{\delta}, \\
V(0) & =\frac{\beta V_{0}}{\delta} .
\end{aligned}
$$

2.1. Equilibrium Points. In order to discover the model's equilibrium points, all the equations of model (4) are put equal to zero. In such a way, two states are achieved. The first state is the equilibrium point at which the cells and tumors tend to zero [37]. This shows the success of virotherapy treatment. The point for equilibrium is $E_{0}=(0,0,0)$. The second state is partial treatment, i.e., the cells with the existence of viruses such as $E^{*}=(h / 1-g, f h / 1-g, f)$. The existence of $E^{*}$ corresponds with $g<1$, while $E_{0}$ exists always. Moreover, the system is dealt as

$$
\begin{array}{r}
I V=0, \\
I V-U=0, \\
U-g I V-h V=0 .
\end{array}
$$

In the abovementioned case, we suppose $f=0$. From equation (6), we have that $I=0$ or $V=0$. If $I=0$, we can get $E_{0}=(0,0,0)$. When $V=0$, from equation (7), we obtain $U=0$, which gives from equation (8) $-g I V=0$ for all $I$. This concludes that $I$ is arbitrary and, hence, for any $I_{1}$, the equilibrium point is $E_{1}=\left(I_{1}, 0,0\right)$. For the equilibrium point, the initial condition is a main factor [12]; it tends the equilibrium to $E_{0}$ or $E_{1}$.

$$
\begin{gathered}
f I-I V=0, \\
I V-U=0, \\
U-g I V=0 .
\end{gathered}
$$

Now, in this case, $h=0$ is considered for obtaining the equilibrium point. From equation (9), we have $I=0$ or $V=f$. By $I=0$, equation (10) gives $U=0$, and for the value of $U$, equation (11) implies $-g I V=0$ for all values of $V$. So, $V$ is arbitrary. Thus, the equilibrium point becomes $E_{2}=\left(0,0, V_{2}\right)$. In a similar way, for $V=f$, the equilibrium point tends to $E_{3}=(0,0, f)$ as a spacial case of $E_{2}$. The initial condition affects the size of viruses [8].

2.2. Stability Analysis. In this section, local and global stability of the equilibrium point are investigated. For investigation of local stability, the linearization method [39] is implemented, and for global stability, Lyapunov function [40] is implemented. First, the local stability of $E_{0}$ and $E^{*}$ is investigated by implementing the linearization method. System (4) will be locally stable if $f>0$, and the equilibrium point $E_{0}$ is an unstable saddle point. So, the Jacobian of model (4) at $E_{0}$ can be written as 


$$
J\left(E_{0}\right)=\left[\begin{array}{ccc}
f & 0 & 0 \\
0 & -1 & 0 \\
0 & 1 & -h
\end{array}\right] .
$$

By solving, the eigenvalues of $J\left(E_{0}\right)$ are

$$
\begin{aligned}
& \lambda_{1}=f, \\
& \lambda_{2}=-1, \\
& \lambda_{3}=-h .
\end{aligned}
$$

Hence, $\lambda_{2}$ and $\lambda_{3}$ are nonpositive, while $\lambda_{1}$ is positive since $f>0$. Thus, for $f>0, E_{0}$ is an unstable saddle point.

Now, for investigation of global stability, the Lyapunov function is implemented. The equilibrium point $E_{1}$ will be globally stable if $I_{1} \leq h / 1-g^{\prime}$. By considering the Lyapunov function,

$$
L(I, U, V)=(1-g)\left(I-I_{1}-I_{1} \ln \left(\frac{I}{I_{1}}\right)\right)+U+V .
$$

It is clearly observed that $L(I, U, V)$ is positive definite because $g$ is negative. Analysing the derivative of $L$ with the positive solutions of model (1), we get

$$
\begin{aligned}
\frac{\mathrm{d} L}{\mathrm{~d} t} & =(1-g)\left(1-\frac{I_{1}}{I}\right)(-I V)+I V-g I V-h V, \\
& =(1-g) I_{1} V+I V-h V, \\
& =\left(I_{1}(1-g)-h\right) V .
\end{aligned}
$$
stable.

As $I_{1} \leq h / 1-g, \mathrm{~d} L / \mathrm{d} t \leq 0$. This shows that $E_{1}$ is globally

\section{Proposed Methodology}

The proposed scheme for the solution of the mathematical model of cancer virotherapy (CVRT) is presented here. Firstly, the mathematical model of CVRT is designed in terms of an Artificial neural network (ANN). Secondly, based on mean squared error, fitness function is constructed, and lastly, the proposed optimization scheme is described.

3.1. Mathematical Model of CVRT. The artificial neural network-based model is designed for the solution of CVRT. The activation function is used, given in equation (19), for the approximation of the solution of CVRT. The model proceeds as

$$
\left.\begin{array}{l}
\widehat{I}(t)=\sum_{i=1}^{k} a_{I i} \theta\left(w_{I i}(t)+b_{I i}\right) \\
\widehat{I}^{\prime}(t)=\sum_{i=1}^{k} a_{I i} \theta^{\prime}\left(w_{I i}(t)+b_{I i}\right) \\
\widehat{I}^{\prime \prime}(t)=\sum_{i=1}^{k} a_{I i} \theta^{\prime \prime}\left(w_{I i}(t)+b_{I i}\right) \\
\vdots \\
\widehat{I}^{(n)}(t)=\sum_{i=1}^{k} a_{I i} \theta^{(n)}\left(w_{I i}(t)+b_{I i}\right)
\end{array}\right\},
$$

Equations (16)-(18) represent the artificial neural network-based model of CVRT. Here, $\theta$ represents the logsigmoid activation function defined in equation (19). The $\log$-sigmoid function gives the values between 0 and 1 . It has a smooth gradient preventing jumps which helps the network in a rapid convergence. It normalizes the output for each neuron. It can show the probability of the output of the network. The ANN form of the activation function and its derivative is given in equations (20) and (21), respectively.

$$
\begin{aligned}
\theta(t) & =\frac{1}{1+e^{-t},} \\
\widehat{\theta}(t) & =\sum_{i=1}^{k} a_{i}\left(\frac{1}{1+e^{-\left(w_{i} t+b_{i}\right)}}\right),
\end{aligned}
$$




$$
\widehat{\theta}^{\prime}(t)=\sum_{i=1}^{k} a_{i} w_{i}\left(\frac{e^{-\left(w_{i} t+b_{i}\right)}}{\left(1+e^{-\left(w_{i} t+b_{i}\right)}\right)^{2}}\right) .
$$

Equation (20) is based on variables known as weights, represented by set $W$, where $W=\left[\mathbf{a}_{i}, \mathbf{w}_{i}, \mathbf{b}_{i}\right]$ and its constituents $\mathbf{a}_{i}=a_{1}, a_{2}, a_{3}, \ldots a_{k}, \mathbf{w}_{i}=w_{1}, w_{2}, w_{3}, \ldots w_{k}$, and $\mathbf{b}_{i}=b_{1}, b_{2}, b_{3}, \ldots b_{k}$.

3.2. Fitness Function for the Model of CVRT. The fitness function for the mathematical model of CVRT is based on the mean square error of the differential equation of the model, given in equation (4), and associated initial conditions, given in equation (5). The fitness function is defined as follows:

$$
E=E_{I}+E_{U}+E_{V}+E_{c},
$$

where, $E_{I}, E_{U}$, and $E_{V}$ are errors associated with differential equations of model (4) written as

$$
\begin{aligned}
E_{I} & =\frac{1}{N} \sum_{m=1}^{N}\left(\frac{\widehat{\mathrm{d} I_{m}}}{\mathrm{~d} t}-f \widehat{I}_{m}+\widehat{I}_{m} \widehat{V}_{m}\right)^{2}, \\
E_{U} & =\frac{1}{N} \sum_{m=1}^{N}\left(\frac{\widehat{\mathrm{d} U_{m}}}{\mathrm{~d} t}-\widehat{I}_{m} \widehat{V}_{m}+\widehat{U}_{m}\right)^{2}, \\
E_{V} & =\frac{1}{N} \sum_{m=1}^{N}\left(\frac{\widehat{\mathrm{d} V_{m}}}{\mathrm{~d} t}-\widehat{U}_{m}+g \widehat{I}_{m} \widehat{V}_{m}+h \widehat{V}_{m}\right)^{2} .
\end{aligned}
$$

Also, $E_{c}$ is associated with initial conditions given as

$$
E_{c}=\frac{1}{3}\left(\left(\widehat{I}-I_{0}\right)^{2}+\left(\widehat{U}-U_{0}\right)^{2}+\left(\widehat{V}-V_{0}\right)^{2}\right) \text {. }
$$

3.3. Optimization Scheme. The scheme is designed by integrating the unsupervised scheme Sine-Cosine Algorithm (SCA) and the supervised technique Sequential Quadratic Programming (SQP). The SCA was first introduced by Syed Ali [42]. The technique is designed for the solution of optimization problems. Its phenomena are based on trigonometric functions sine and cosine. Normally, the population-based technique generates several solutions that are further modified by utilizing the strength of that technique. For improvement, the set of rules called the kernel of optimization technique is used. The population-based technique is not necessary to get the optimal solution in a single run. However, the population of solutions increases the probability of getting the global optimal solution. Generally, regardless of the difference between stochastic algorithms, the works in two phases are called exploration and exploitation. In the first phase, a highly random set of solutions is generated to find a feasible region. Also, that region is gradually exploited for the best optimal solution. However, the variation is less as compared to the exploration. The following equations are used in SCA for updating the position in both phases:

$$
\begin{aligned}
& X_{i}^{t+1}=X_{i}^{t}+r_{1} \times \sin \left(r_{2}\right) \times\left|r_{3} P_{i}^{t}-X_{i}^{t}\right|, \\
& X_{i}^{t+1}=X_{i}^{t}+r_{1} \times \cos \left(r_{2}\right) \times\left|r_{3} P_{l}^{t}-X_{i_{j}}^{t}\right|,
\end{aligned}
$$

where the position of the current solution in the $i^{\text {th }}$ dimension at the $t^{\text {th }}$ iteration is denoted by $X_{i}^{t}, r_{r} / r_{2} / r_{3}$ are random numbers, and $P_{i}$ indicates the position of the destination point and denotes the absolute value.

Equation (27) with parametric values can be written as

$$
X_{l}^{t+1}= \begin{cases}X_{i}^{t}+r_{1} \times \sin \left(r_{2}\right) \times\left|r_{3} P_{l}^{t}-X_{i}^{t}\right|, & r_{4}<0.5, \\ X_{l}^{t}+r_{1} \times \cos \left(r_{2}\right) \times\left|r_{3} P_{l}^{t}-X_{L_{i}}^{t}\right|, & r_{4} \geq 0.5,\end{cases}
$$

where $r_{4}$ indicates a random value in $[0,1]$. In the abovementioned equation, there are four parameters $r_{1}, r_{2}, r_{3}$, and $r_{4} . r_{1}$ indicates the movement direction which could be feasible or outside that region. Parameter $r_{2}$ describes the distance from the region, that is, how far the direction is toward or outward the target. Parameter $r_{3}$ defines weights for target value, and $r_{4}$ equally operates the sine and cosine constituent of equation (26). This method is integrated with sequential quadratic programming to improve the quality of the generated solution. As the SCA is a global search unsupervised algorithm and based on a random population, some time is stuck in the local search. The SQP is a local search algorithm and supervised method. The generated solution of SCA is used as an initial point for SQP. Sequential quadratic programming is an effective technique to solve nonlinear optimization problems. The SQP has a strong theoretical and computational background due to which it generates promising results. That is why the integration of SQP with SCA improves the local performance of the SCA. The flow chart of the ANN-SCA-SQP procedure is given in Figure 1. The integration of these techniques is based on the strength of the artificial neural network. This gives quality and effective results. The scheme throughout the text is abbreviated as ANN-SCA-SQP algorithm. To validate the performance of the ANN-SCA-SQP algorithm, different performance indicators are implemented.

3.4. Performance Indicators. Statistical performance operators are utilized to validate and evaluate the performance of the proposed methodology, i.e., ANN-SCA-SQP algorithm. The implemented performance indicators are Mean Absolute Deviation (MAD), Root Mean Squared Error (RMSE) and Error in Nash-Sutcliffe Efficiency (ENSE) based on Nash-Sutcliffe (NSE). The performance operators are defined for each variable of the model of CVRT. The definition of performance indicators is given in the following mathematical forms: 


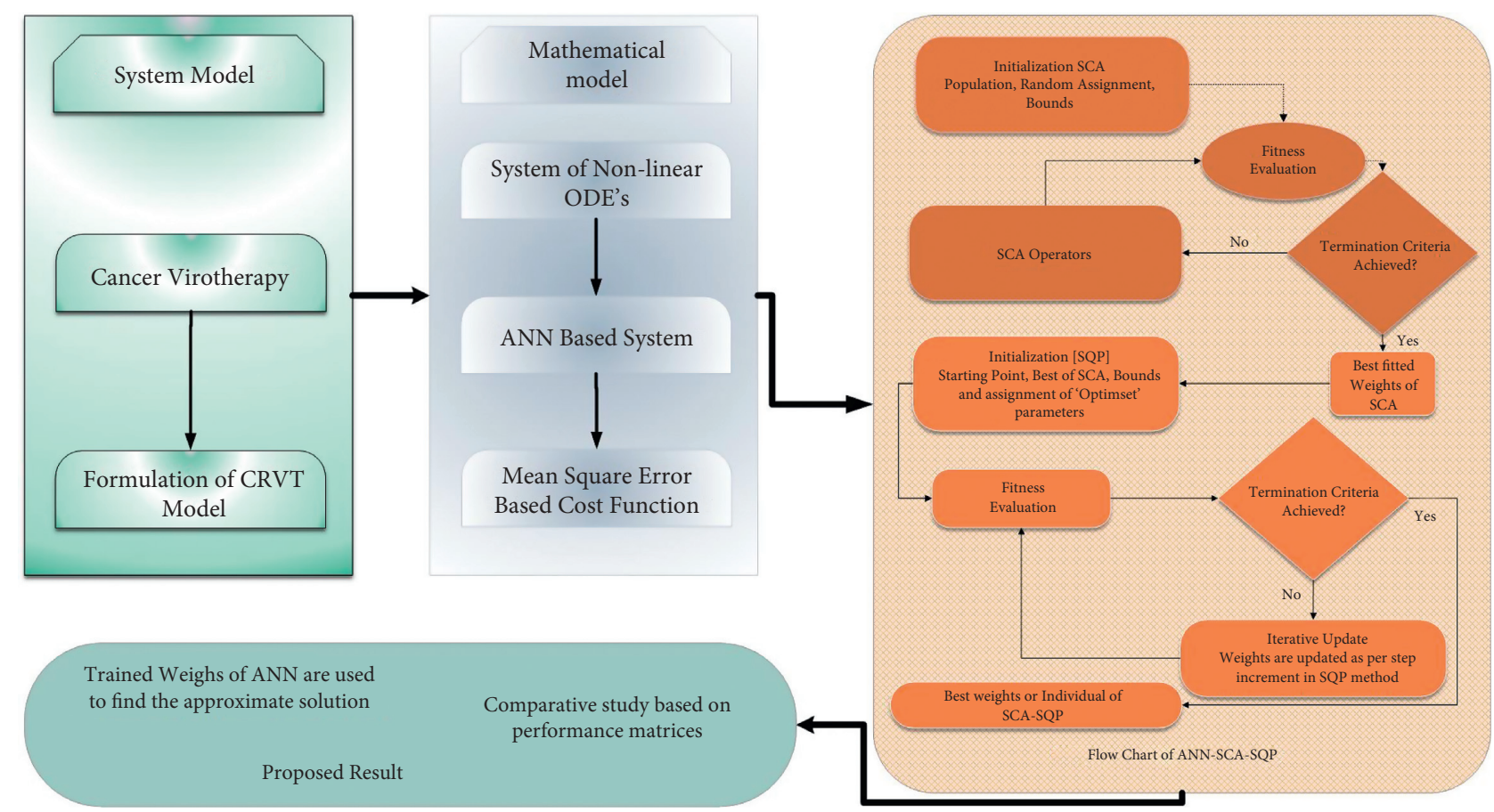

FIGURE 1: The flow chart of the proposed methodology ANN-SCA-SQP. Here, SCA generates a number of random solutions and evaluates it on its fitness; then, it is provided to SQP as an initial point for further refinement. The fittest weights are obtained for the solution of CVRT.

$$
\begin{aligned}
& \left.\left[\begin{array}{lll}
\operatorname{MAD}_{I} & \mathrm{MAD}_{U} & \mathrm{MAD}_{V}
\end{array}\right]=\left[\frac{1}{m} \sum_{i=1}^{m}\left|I_{i}-\widehat{I}_{i}\right| \frac{1}{m} \sum_{i=1}^{m}\left|U_{i}-\widehat{U}_{i}\right| \frac{1}{m} \sum_{i=1}^{m}\left|V_{i}-\widehat{V}_{i}\right|\right]\right],
\end{aligned}
$$

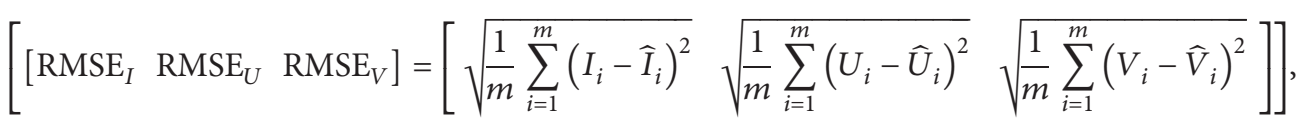

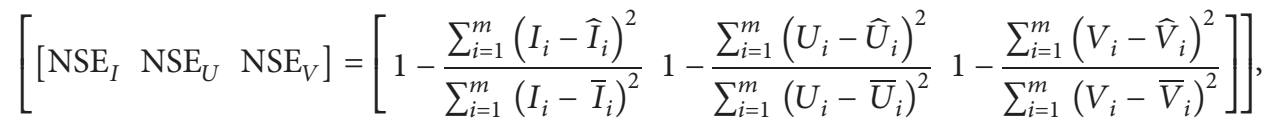$$
\left.\left[\begin{array}{lll}
\mathrm{ENSE}_{I} & \mathrm{ENSE}_{U} & \mathrm{ENSE}_{V}
\end{array}\right]=\left[\left|1-N S E_{I}\right|\left|1-\mathrm{NSE}_{U}\right|\left|1-N S E_{V}\right|\right]\right]
$$

where $m$ denotes the input points, while $I, U$, and $V$ are reference solutions of the CVRT model found through the Runge-Kutta order four technique (RK4) and $\widehat{I}, \widehat{U}$, and $\widehat{V}$ are the approximated solutions of the proposed methodology. The desired value of MAD, RMSE, and ENSE is 0, while the suitable value for NSE is 1 .
Moreover, to verify the quality, efficiency, and effectiveness of the proposed scheme, the global extension of the performance indicator is also utilized. The global version of MAD, RMSE, and ENSE is denoted by GMAD, GRMSE, and GENSE. The definition of global performance operators is as follows:

$$
\begin{aligned}
{\left[\left[\operatorname{GMAD}_{I} \mathrm{GMAD}_{U} \mathrm{GMAD}_{V}\right]\right.} & =\left[\frac{1}{I} \sum_{i=1}^{I}\left(\frac{1}{m} \sum_{k=1}^{m}\left(\left|I_{i}-\widehat{I}_{i}\right|\right)\right)_{i}^{4} \frac{1}{T} \sum_{i=1}^{l}\left(\frac{1}{m} \sum_{k=1}^{m}\left(\left|U_{i}-\widehat{U}_{i}\right|\right)\right)_{T} \sum_{i} \sum_{i=1}^{I}\left(\frac{1}{m} \sum_{K=1}^{m}\left(\left|V_{i}-\widehat{V}_{i}\right|\right)\right)\right] \\
{\left[\operatorname{GRMSE}_{I} \operatorname{GRMSE}_{U} \operatorname{GRMSE}_{V}\right] } & =\left[\frac{1}{T} \sum_{i=1}^{I}\left(\sqrt{\frac{1}{m} \sum_{k=1}^{m}\left(I_{i}-\widehat{I}_{i}\right)^{2}}\right)_{i}^{1} \frac{1}{T} \sum_{i=1}^{I}\left(\sqrt{\frac{1}{m} \sum_{k=1}^{m}\left(U_{i}-\widehat{U}_{i}\right)^{2}}\right)_{i} \frac{1}{T} \sum_{i=1}^{I}\left(\sqrt{\frac{1}{m} \sum_{k=1}^{m}\left(V_{i}-\widehat{V}_{i}\right)^{2}}\right)\right],
\end{aligned}
$$


$\left[\begin{array}{lll}\operatorname{GENSE}_{I} & \operatorname{GENSE}_{U} \quad \mathrm{GENSE}_{V}\end{array}\right]$

$$
=\left[\frac{1}{I} \sum_{i=1}^{I}\left(1-\frac{\sum_{k=1}^{m}\left(I_{i}-\widehat{I}_{i}\right)^{2}}{\sum_{k=1}^{m}\left(I_{i}-\widehat{I}_{i}\right)^{2}}\right)_{i} \frac{1}{I} \sum_{i=1}^{I}\left(1-\frac{\sum_{k=1}^{m}\left(U_{i}-\widehat{U}_{i}\right)^{2}}{\sum_{k=1}^{m}\left(U_{i}-\widehat{U}_{i}\right)^{2}}\right)_{i} \frac{1}{I} \sum_{i=1}^{I}\left(1-\frac{\sum_{k=1}^{m}\left(V_{i}-\widehat{V}_{i}\right)^{2}}{\sum_{k=1}^{m}\left(V_{i}-\widehat{V}_{i}\right)^{2}}\right)_{i}\right] .
$$

In equations (33)-(35), I represents the number of runs. The scheme is executed for multiple runs. The global operators depend on the average values of their respective operators.

\section{Discussion and Results}

In this section, the dynamic characteristic of the CVRT model is given. The discussion on CVRT is split into different problems. The problems depend on the variation of the initial condition and its parameters $f, g$, and $h$. The results are based on 100 execution (runs).

4.1. Problem 1. In this problem, the dynamic characteristic of the CVRT model, given in equation (4), is assessed by taking values of tumor growth ratio $(r)$ to rate of cell lysis $(\delta)$ $f=0$, the inverse of burst size parameter $g=0.02$, and the viral decay $(\alpha)$ ratio to rate of cell lysis $(\delta) h=0.15$. The problem is executed multiple times. The fitness function with 10 input points can be updated as

$$
\begin{aligned}
E= & \left(\frac { 1 } { 1 0 } \sum _ { m = 1 } ^ { 1 0 } \left(\left(\frac{\mathrm{d} \widehat{I}_{m}}{\mathrm{~d} t}-0 . \widehat{I}_{m}+\widehat{I}_{m} \widehat{V}_{m}\right)^{2}+\left(\frac{\mathrm{d} \widehat{U}_{m}}{\mathrm{~d} t}-\widehat{I}_{m} \widehat{V}_{m}+\widehat{U}_{m}\right)^{2}\right.\right. \\
& \left.+\left(\frac{\mathrm{d} \widehat{V}_{m}}{\mathrm{~d} t}-\widehat{U}_{m}+0.02 \widehat{I}_{m} \widehat{V}_{m}+0.15 \widehat{V}_{m}\right)^{2}\right) \\
& \left.+\frac{1}{3}\left(\left(\widehat{I}_{0}-0.6\right)^{2}+\widehat{U}_{0}^{2}+\left(\widehat{V}_{0}-0.5\right)^{2}\right)\right) .
\end{aligned}
$$

Also, the associated initial conditions are $I(0)=0.6, U(0)=0$, and $V(0)=0.5$.

The proposed scheme ANN-SQP-SCA is implemented to optimize the fitness function. The solution is compared with the solution of Range-Kutta order 4 (RK4). The set of weights for each variable, $I(t), U(t)$, and $V(t)$, is found with fitness between $10^{-07}$ to $10^{-12}$. The fitness values are shown graphically in Figure 2(a). The result of RK4 is obtained by implementing Matlab's built-in function Ode45. The solutions are graphically compared with RK4 given in Figure 3(a). Each variable $I(t), U(t)$, and $V(t)$ is drawn separately for 100 independent runs in Figures 3(c)-3(e). The obtained variable, known as weight, is also plotted in Figure 3(b). The weights are plotted with a 3D bar graph. The fitness value of the problem is evaluated through statistical operators minimum (MIN), maximum (MAX), and mean (MEAN). Data for statistical operators are given in Table 2.

The trained weights used in equations (22)-(25) to get the solution for the CVRT model are given as

$$
\begin{aligned}
& \widehat{I}(t)=\frac{-0.0191}{1+e^{-(0.5764 t+0.1135)}}+\cdots+\frac{0.8319}{1+e^{-(0.5268 t+0.715)}}, \\
& \widehat{U}(t)=\frac{-1.7428}{1+e^{-(-2.0548 t-0.2668)}}+\cdots+\frac{-0.1394}{1+e^{-(-0.3252 t-2.3175)}}, \\
& \widehat{V}(t)=\frac{0.4388}{1+e^{-(-1.5128 t-0.9588)}}+\cdots+\frac{0.1590}{1+e^{-(-0.1085 t-2.2570)}} .
\end{aligned}
$$

The full form of a solution in (37) is given in Appendix with 14 decimal places to avoid any roundoff error. The overlapping of solutions with the results of RK4 shows the convergence of the proposed scheme. The solution is obtained for $t \in[0,1]$ with step size 0.01 , which gives 101 points. The small step size is taken to verify small variation in the solution.

From Figure 3(a), it can be seen that, initially, with the increase in viruses $(V(t))$, the uninfected cells $(I(t))$ also increase. It can be observed that, with the passage of time, all the variables move toward equilibrium point $(0,0,0)$. The initial condition or stage of cancer is highly effective in the cancer virotherapy.

4.2. Problem 2. This problem is taken for values of tumor growth ratio $(r)$ to rate of cell lysis $(\delta) f=0$, the inverse of burst size parameter $g=0.02$, and the viral decay $(\alpha)$ ratio to rate of cell lysis $(\delta) h=0.15$. The fitness function for this problem can be updated as

$$
E=\left(\begin{array}{c}
\frac{1}{10} \sum_{m=1}^{10}\left(\left(\frac{\mathrm{d} \widehat{I}_{m}}{\mathrm{~d} t}-0 . \widehat{I}_{m}+\widehat{I}_{m} \widehat{V}_{m}\right)^{2}+\left(\frac{\mathrm{d} \widehat{U}_{m}}{\mathrm{~d} t}-\widehat{I}_{m} \widehat{V}_{m}+\widehat{U}_{m}\right)^{2}+\left(\frac{\mathrm{d} \widehat{V}_{m}}{\mathrm{~d} t}-\widehat{U}_{m}+0.02 \widehat{I}_{m} \widehat{V}_{m}+0.15 \widehat{V}_{m}\right)^{2}\right) \\
+\frac{1}{3}\left(\left(\widehat{I}_{0}-0.4\right)^{2}+\widehat{U}_{0}^{2}+\left(\widehat{V}_{0}-0.1\right)^{2}\right)
\end{array}\right) .
$$




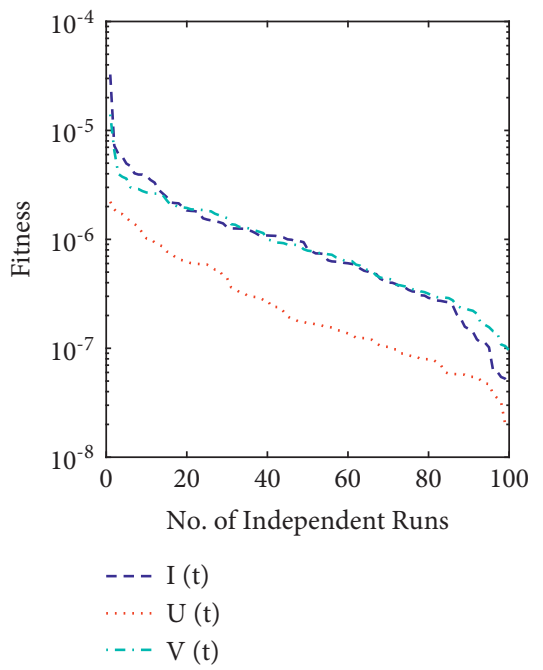

(a)

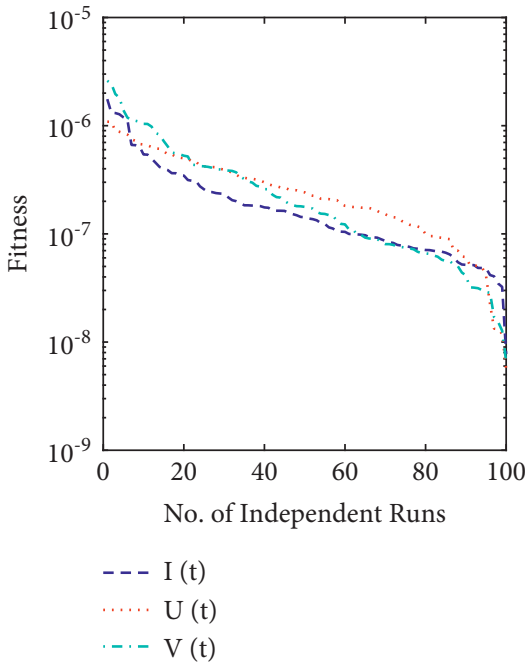

(b)

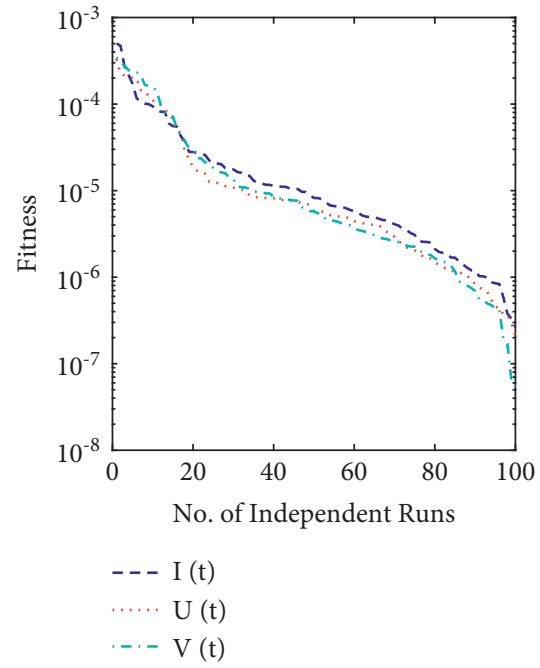

(c)

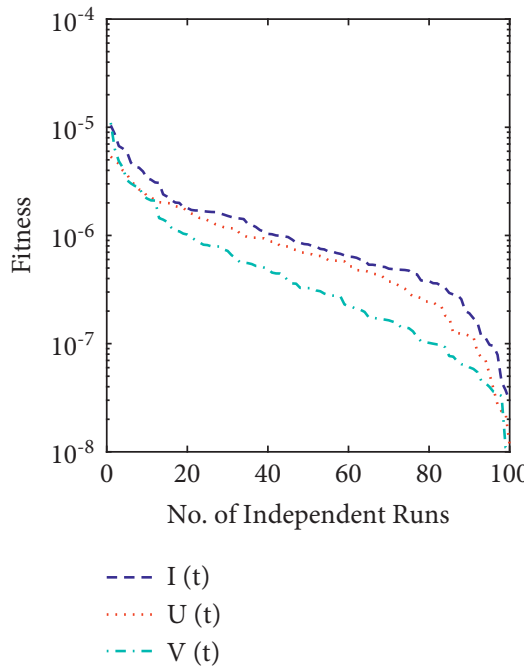

(d)

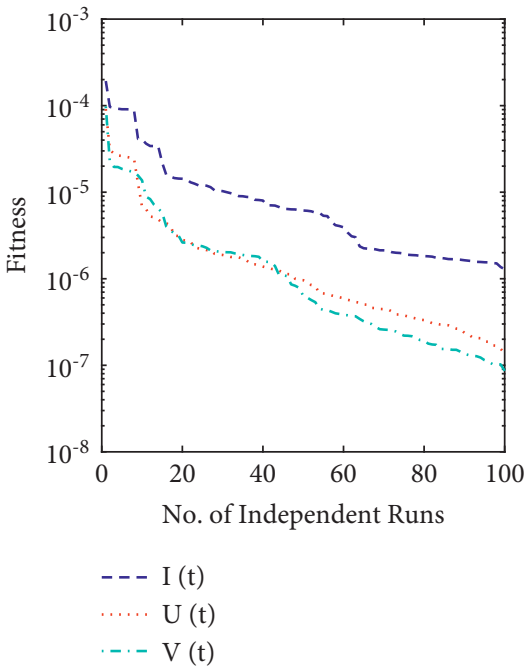

(e)

Figure 2: Fitness graphs of all the problems. (a) Fitness of problem 1, (b) fitness of problem 2, (c) fitness of problem 3, (d) fitness of problem 4 , and (e) ditness of problem 5.

The associated conditions are $I(0)=0.4, U(0)=0$, and $V(0)=0.1$.

The proposed scheme is, as discussed in Section 3, used to solve the problem. The proposed scheme ANN-SQP-SCA is implemented to optimize the fitness function. The solution is compared with the solution of Range-Kutta order 4 (RK4). The set of weights for each variable, $I(t), U(t)$, and $V(t)$, is found with fitness $10^{-9}$ to $10^{-13}$. The fitness values are shown graphically in Figure 2(b). The result of RK4 is obtained by implementing Matlab's built-in function Ode45. The solutions are graphically compared with RK4 given in Figure 4(a). Each variable $I(t), U(t)$, and $V(t)$ is drawn separately for 100 independent runs in Figures 4(c)-4(e). The obtained variable, known as weight, is also plotted in Figure 4(b). The weights are plotted with a 3D bar graph. The fitness value of the problem is evaluated through statistical operators minimum (MIN), maximum (MAX), and mean (MEAN). Data for statistical operators are given in Table 3.
The trained weights are used to get a solution for CVRT model. The obtained set of the weight of ANN is drawn graphically. By putting weights in equations (22)-(25), the solution for each variable should be obtained as

$$
\begin{aligned}
& \widehat{I}(t)=\frac{-0.0060}{1+e^{-(0.2987 t+0.0137)}}+\cdots+\frac{0.2378}{1+e^{-(0.0701 t+0.0006)}}, \\
& \widehat{U}(t)=\frac{-1.8494}{1+e^{-(0.4333 t-2.2236)}}+\cdots+\frac{-0.3929}{1+e^{-(-2.0398 t+0.2293)}}, \\
& \widehat{V}(t)=\frac{0.5100}{1+e^{-(-0.4642 t-0.5303)}}+\cdots+\frac{-0.2720}{1+e^{-(-1.2262 t+0.0342)}} .
\end{aligned}
$$

The full form of (39) is given in Appendix with 14 decimal places to avoid roundoff issues. The set of weights is plotted with a 3-dimensional bar graph. The overlapping of solutions with the results of RK4 shows the convergence of the proposed scheme. The solution is obtained for $t \in[0,1]$ 


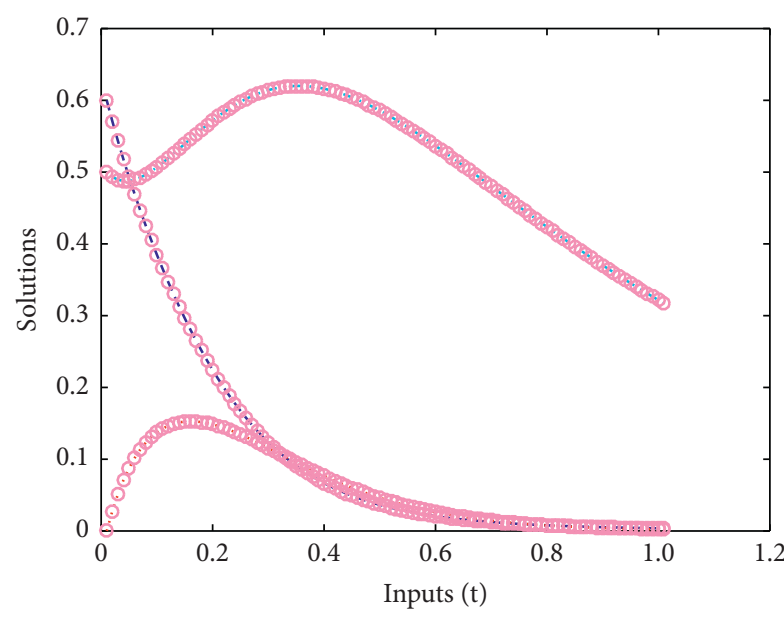
- - I (t)
$\mathrm{U}(\mathrm{t})$
...- V (t)
o Numerical

(a)

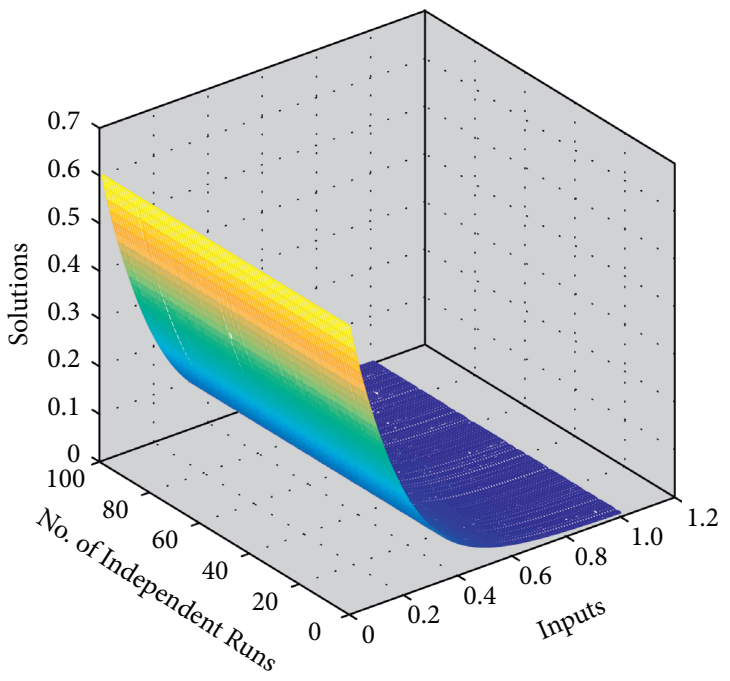

$\square \mathrm{I}(\mathrm{t})$

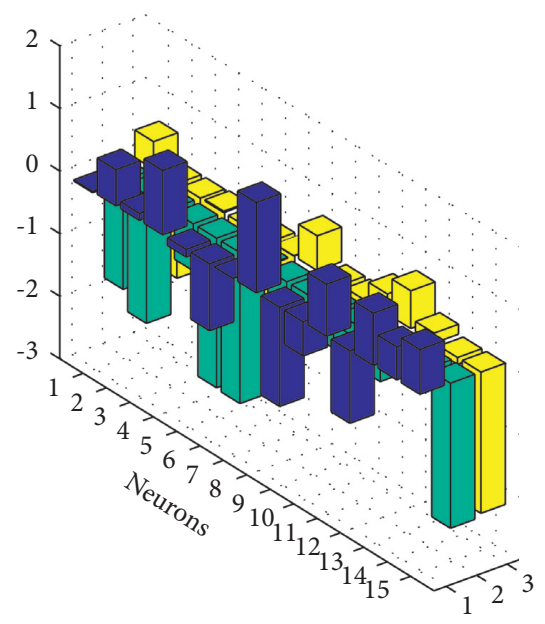

(b)

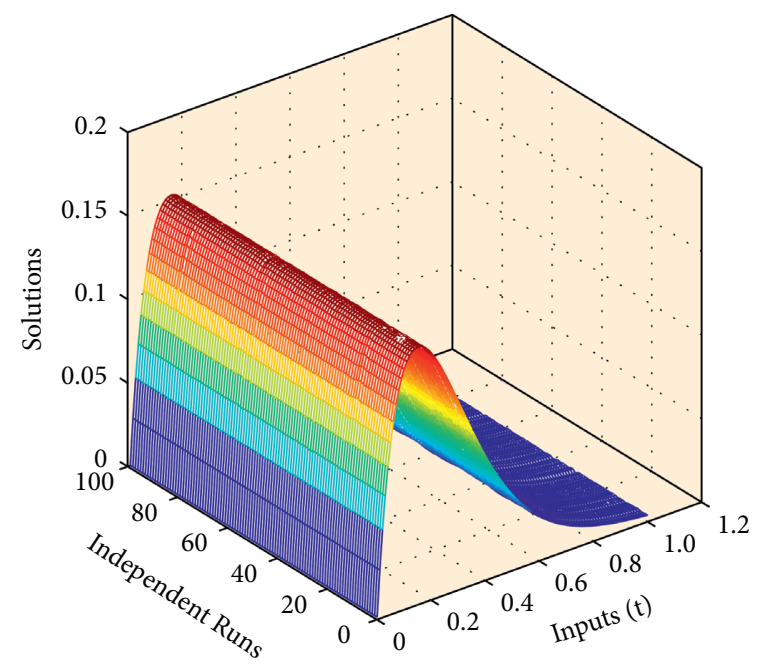

$\square \mathrm{U}(\mathrm{t})$

(c)

(d)

FIgURE 3: Continued. 


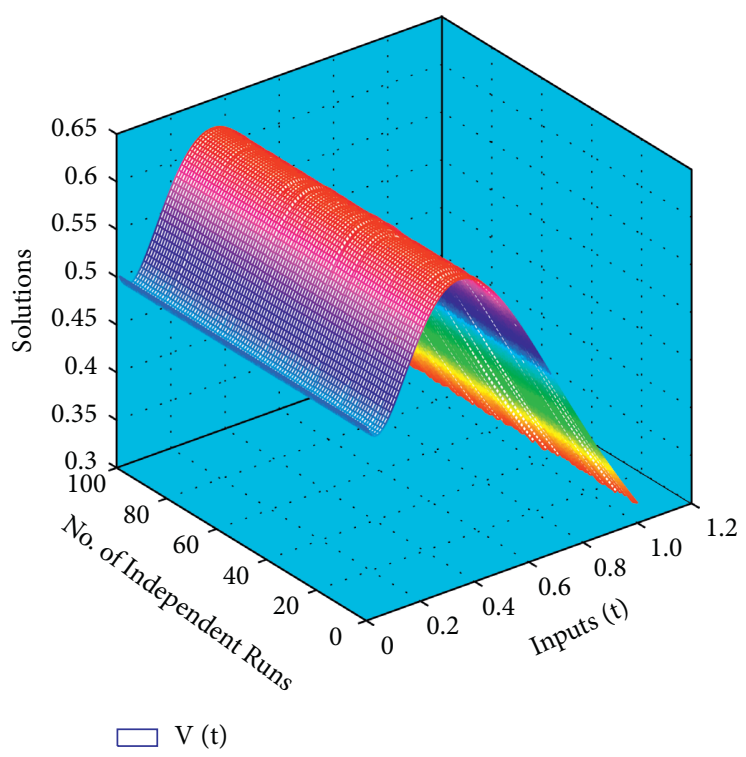

(e)

Figure 3: Problem 1: graphical evaluation tumor growth ratio $(r)$ to rate of cell lysis $(\delta) f=0$, the inverse of burst size parameter $g=0.02$, and the viral decay $(\alpha)$ ratio to rate of cell lysis $(\delta) h=0.15$. (a) Solution for $f=0, g=0.02$, and $h=0.15$ with initial conditions $I(0)=0.6, U$ $(0)=0$, and $V(0)=0.5$, (b) weights obtained by ANN-SCA-SQP for CVRT, (c) solutions of 100 independent runs of uninfected cells, $I(t)$, (d) solutions of 100 independent runs of infected cells, $U(t)$, and (e) solutions of 100 independent runs of free viruses, $V(t)$.

with step size 0.01 , which gives 101 points. The small step size is taken to verify small variation in the solution.

Figure 4(a) shows that, with same values of $f, g$, and $h$ but different initial conditions, the infected cells $(I(t))$ are less than 0.05 and rapidly decrease with passage of time.
4.3. Problem 3. In this problem, the model is discussed for values of tumor growth ratio $(r)$ to rate of cell lysis $(\delta)$ $f=0.36$, the inverse of burst size parameter $g=0.02$, and the viral decay $(\alpha)$ ratio to rate of cell lysis $(\delta) h=0$. For these values, the fitness function is updated as

$$
E=\left(\begin{array}{r}
\frac{1}{10} \sum_{m=1}^{10}\left(\left(\frac{\mathrm{d} \widehat{I}_{m}}{\mathrm{~d} t}-0.36 \widehat{I}_{m}+\widehat{I}_{m} \widehat{V}_{m}\right)^{2}+\left(\frac{\mathrm{d} \widehat{U}_{m}}{\mathrm{~d} t}-\widehat{I}_{m} \widehat{V}_{m}+\widehat{U}_{m}\right)^{2}+\left(\frac{\mathrm{d} \widehat{V}_{m}}{\mathrm{~d} t}-\widehat{U}_{m}+0.02 \widehat{I}_{m} \widehat{V}_{m}+0 . \widehat{V}_{m}\right)^{2}\right) \\
+\frac{1}{3}\left(\left(\widehat{I}_{0}-0.4\right)^{2}+\widehat{U}_{0}^{2}+\left(\widehat{V}_{0}-0.1\right)^{2}\right)
\end{array}\right.
$$

Associated condition are $I(0)=0.4, U(0)=0$, and $V(0)=0.1$.

The proposed scheme ANN-SQP-SCA is implemented to optimize the fitness function. The solution is compared with the solution of Range-Kutta order 4 (RK4). The set of weights for each variable, $x, y$, and $v$, is found with fitness $10^{-09}$ and $10^{-12}$. The fitness values are shown graphically in Figure 2 . The result of RK4 is obtained by implementing Matlab's built-in function Ode45. The solutions are graphically compared with RK4 given in Figure 5(a). Each variable $I(t), U(t)$, and $V(t)$ is drawn separately for 100 independent runs in Figures 5(c)5(e). The obtained variable, known as weight, is also plotted in Figure 5(b). The weights are plotted with a 3D bar graph. The fitness value of the problem is evaluated through statistical operators minimum (MIN), maximum (MAX), and mean (MEAN). Data for statistical operators are given in Table 4.

The trained weights are used in equations (22)-(25) to get the solution for the CVRT model as follows:

$$
\begin{aligned}
\widehat{I}(t) & =\frac{0.0001}{1+e^{-(2.7968 t+3.8980)}}+\cdots+\frac{3.3697}{1+e^{-(1.7235 t-1.2550)}} \\
\widehat{U}(t) & =\frac{0.9174}{1+e^{-(-0.5872 t-0.9386)}}+\cdots+\frac{-0.2933}{1+e^{-(0.8764 t-0.6047)}} \\
\widehat{V}(t) & =\frac{0.2015}{1+e^{-(3.0810 t+5.4546)}}+\cdots+\frac{0.1802}{1+e^{-(-5.5191 t+0.2220)}}
\end{aligned}
$$

The full form of a solution in equation (41) is given in Appendix with 14 decimal places to avoid any roundoff error. The overlapping of solutions with the results of RK4 shows the convergence of the proposed scheme. The solution is obtained for $t \in[0,1]$ with step size 0.01 , which gives 101 points. The small step size is taken to verify small variation in the solution.

As shown in Figure 5(a), if the tumor growth ratio $(r)$ to rate of cell lysis $(\delta), f$, with a decrease in the viral decay $(\alpha)$ ratio to rate of cell lysis $(\delta), h$, the viruses increase very rapidly but the infected cell decreases with passage of time. It 


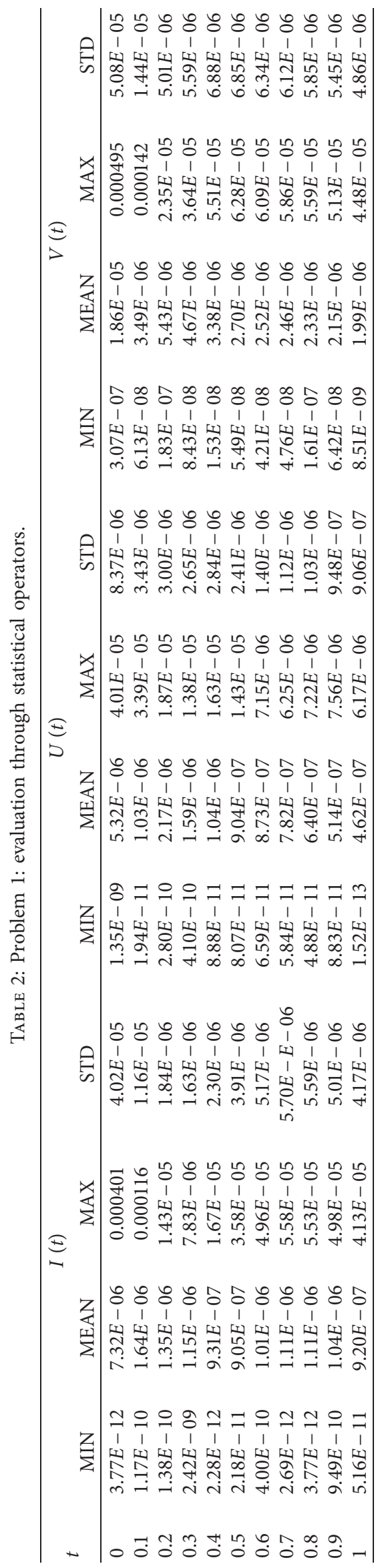




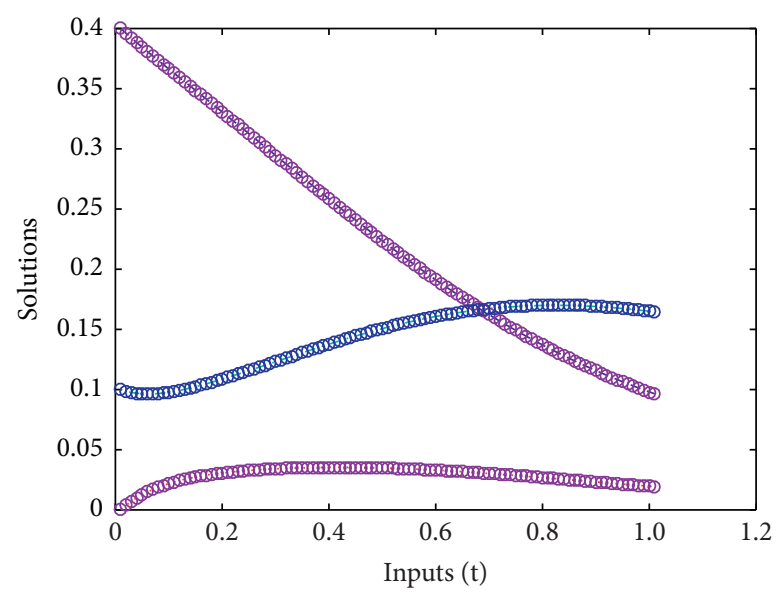
-. - I (t)
$\mathrm{U}(\mathrm{t})$
$\ldots \mathrm{V}(\mathrm{t})$
- Numerical

(a)

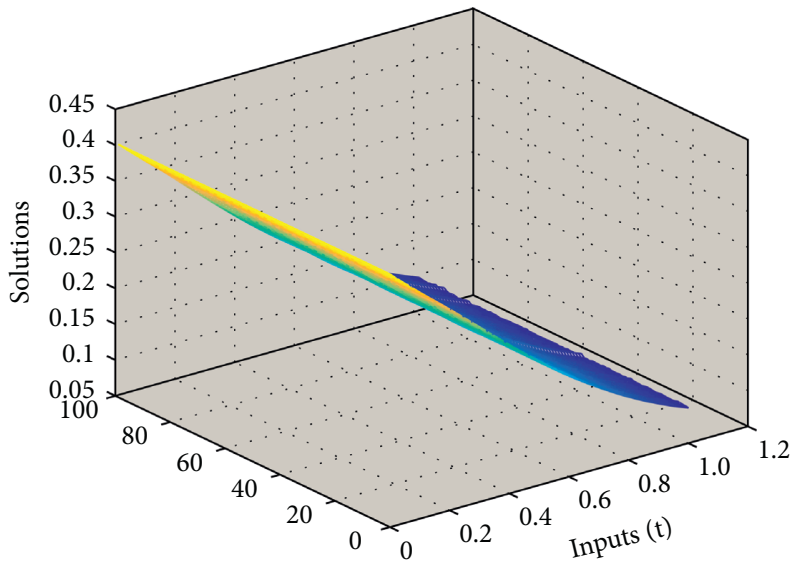

$\square \mathrm{I}(\mathrm{t})$

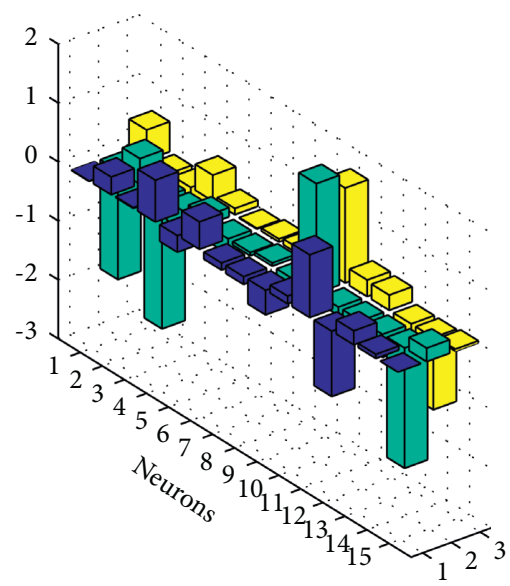

(b)

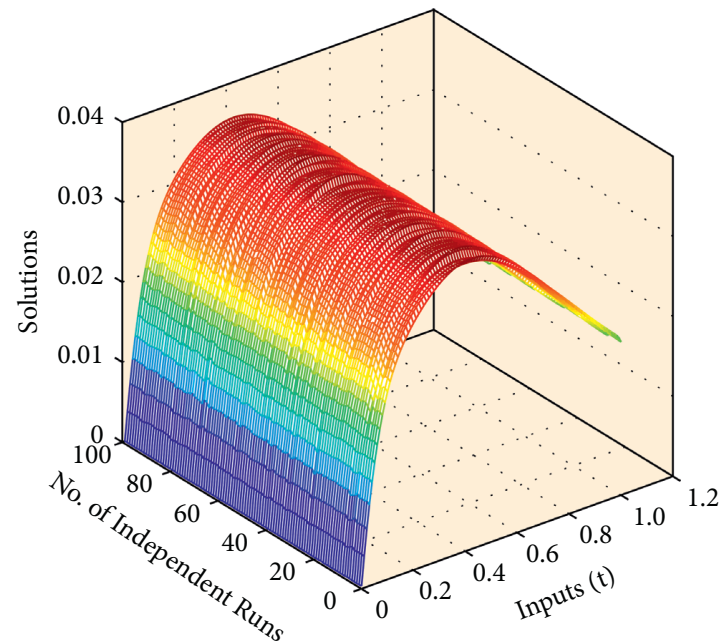

$\mathrm{U}(\mathrm{t})$

(d)

(c)

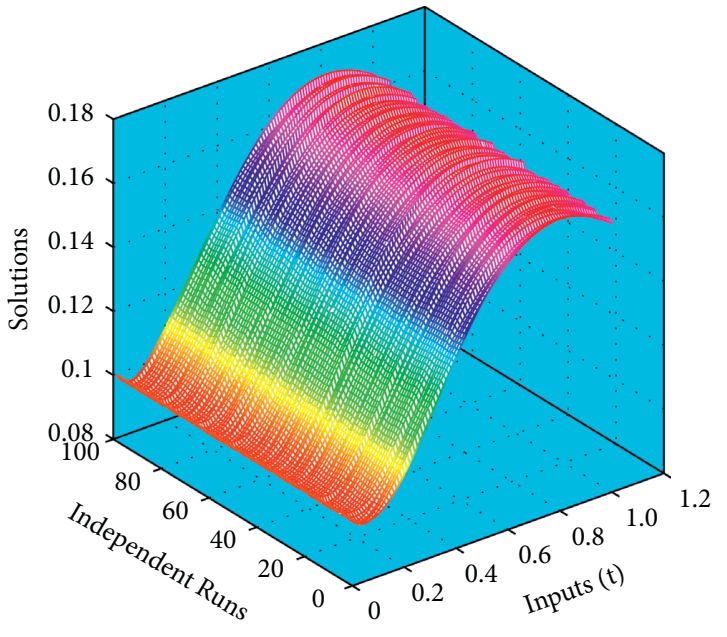

$\mathrm{V}(\mathrm{t})$

(e)

Figure 4: Problem 2: graphical evaluation for tumor growth ratio $(r)$ to rate of cell lysis $(\delta) f=0$, the inverse of burst size parameter $g=0.02$, and the viral decay $(\alpha)$ ratio to rate of cell lysis $(\delta) h=0.15$. (a) Solution for $f=0, g=0.02$, and $h=0.15$ with initial conditions $I(0)=$ $0.4, U(0)=0$, and $V(0)=0.1$, (b) weights obtained by ANN-SCA-SQP for CVRT, (c) solutions of 100 independent runs of $I(t)$, (d) solutions of 100 independent runs of $U(t)$, and (e) solutions of 100 independent runs of $V(t)$. 


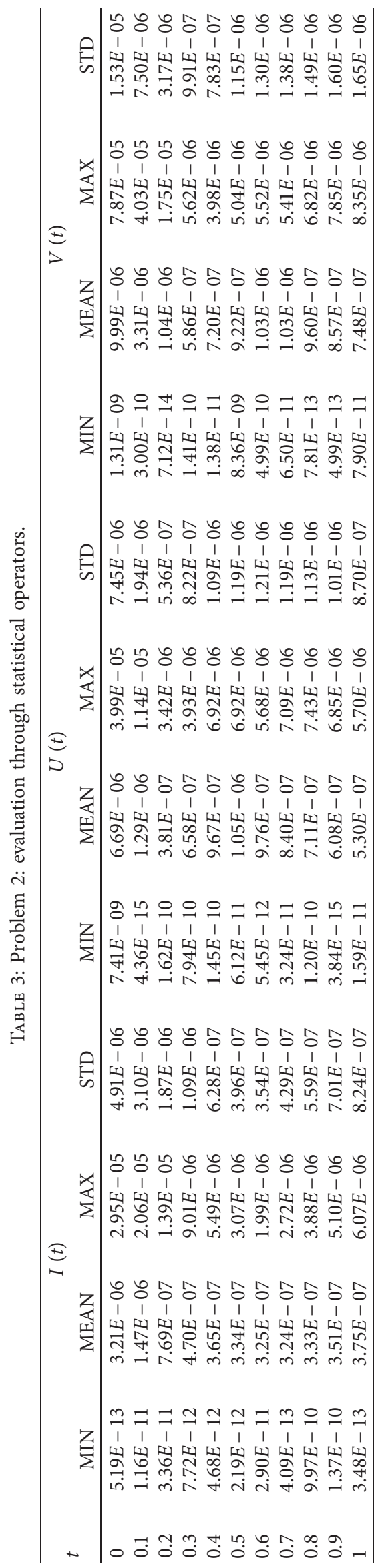




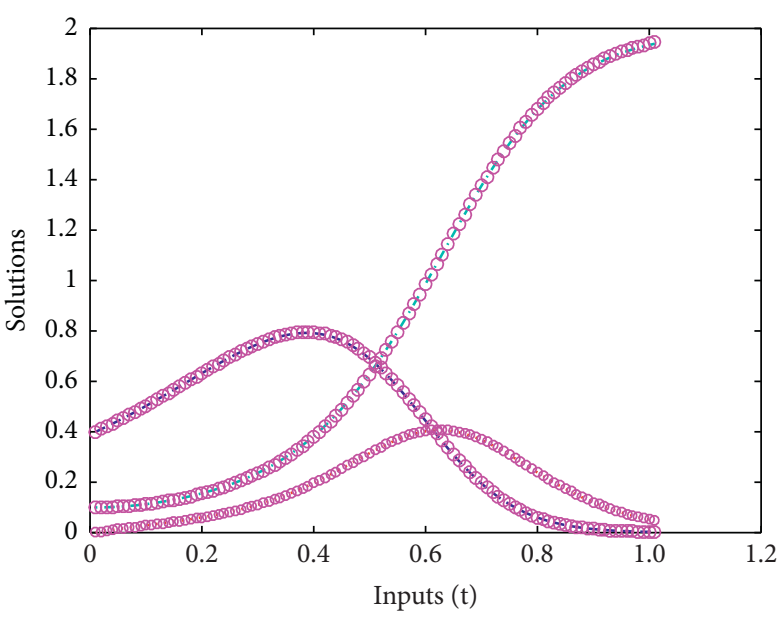

$$
\begin{array}{ll}
--- & I(t) \\
\cdots & U(t)
\end{array}
$$

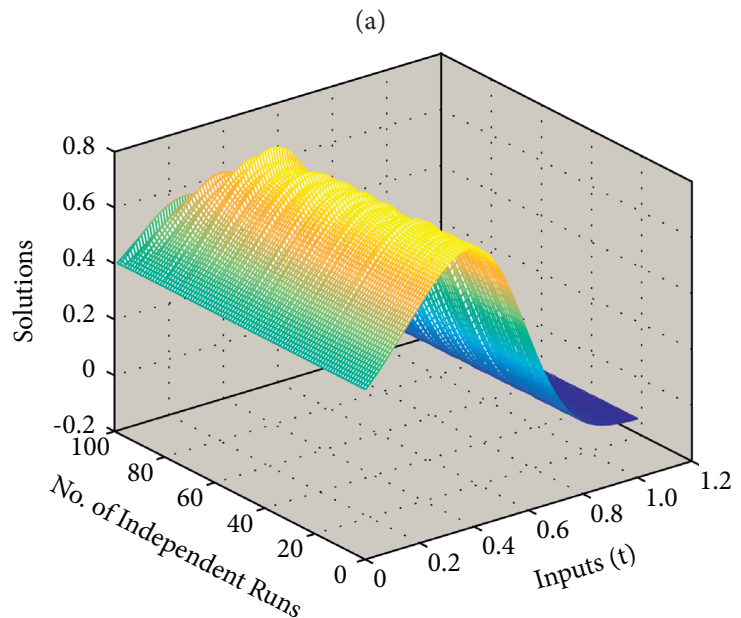

$\square \mathrm{I}(\mathrm{t})$

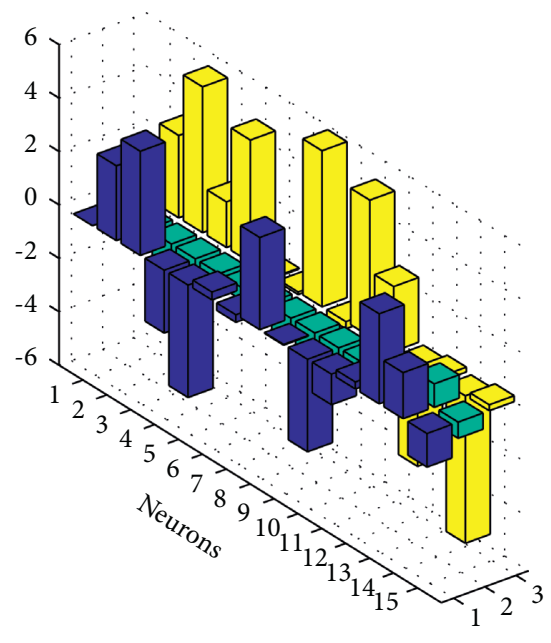

(b)

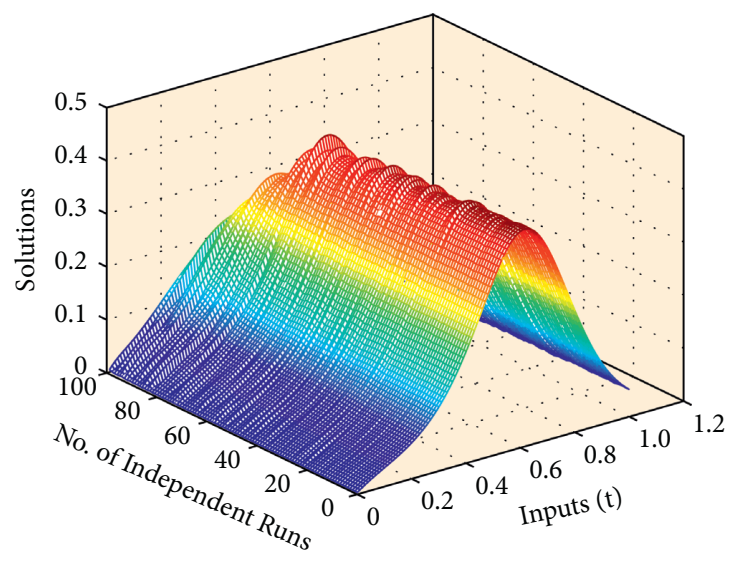

$\mathrm{U}(\mathrm{t})$

(c)

(d)

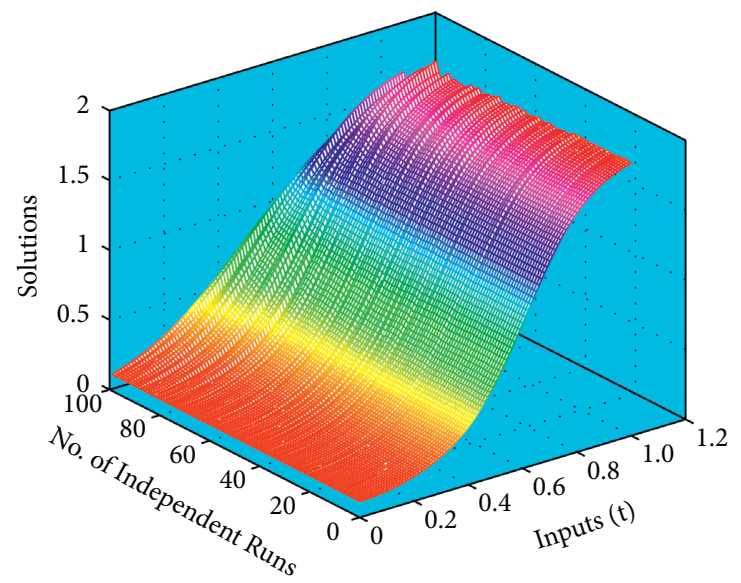

$\square \mathrm{V}(\mathrm{t})$

(e)

Figure 5: Problem 3: graphical evaluation for tumor growth ratio $(r)$ to rate of cell lysis $(\delta) f=0.36$, the inverse of burst size parameter $g=0.02$, and the viral decay $(\alpha)$ ratio to rate of cell lysis $(\delta) h=0$. (a) Solution $f=0.36, g=0.02$, and $h=0$ with conditions $I(0)=0.4, U(0)=$ 0 , and $V(0)=0.1$, (b) weights obtained by ANN-SCA-SQP for CVRT, (c) solutions of 100 independent runs of $I(t)$, (d) solutions of 100 independent runs of $U(t)$, and (e) solutions of 100 independent runs of $V(t)$. 


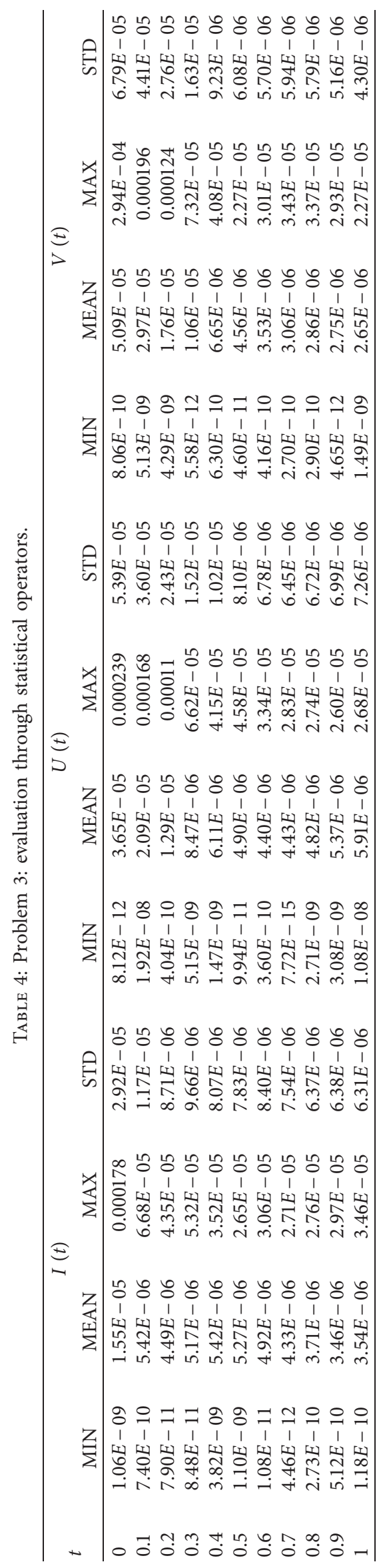


shows the stability of equilibrium point $(0,0, f)$; that is, with the existence of viruses, the infected cells decrease.

4.4. Problem 4. The problem shows dynamics of the CVRT model for tumor growth ratio $(r)$ to rate of cell lysis $(\delta)$ $f=0.36$, the inverse of burst size parameter $g=0.02$, and the viral decay $(\alpha)$ ratio to rate of cell lysis $(\delta) h=0$. These values update the system as

$$
E=\left(\begin{array}{r}
\frac{1}{10} \sum_{m=1}^{10}\left(\left(\frac{\mathrm{d} \widehat{I}_{m}}{\mathrm{~d} t}-0.36 \widehat{I}_{m}+\widehat{I}_{m} \widehat{V}_{m}\right)^{2}+\left(\frac{\mathrm{d} \widehat{U}_{m}}{\mathrm{~d} t}-\widehat{I}_{m} \widehat{V}_{m}+\widehat{U}_{m}\right)^{2}+\left(\frac{\mathrm{d} \widehat{V}_{m}}{\mathrm{~d} t}-\widehat{U}_{m}+0.02 \widehat{I}_{m} \widehat{V}_{m}+0 . \widehat{V}_{m}\right)^{2}\right) \\
+\frac{1}{3}\left(\left(\widehat{I}_{0}-0.4\right)^{2}+\widehat{U}_{0}^{2}+\left(\widehat{V}_{0}-0.5\right)^{2}\right)
\end{array}\right)
$$

Associated condition are $I(0)=0.4, U(0)=0$, and $V(0)=0.5$.

The proposed scheme ANN-SQP-SCA is implemented to optimize the fitness function. The solution is compared with the solution of Range-Kutta order 4 (RK4). The set of weights for each variable, $I(t), U(t)$, and $V(t)$, is found with fitness $10^{-09}$ and $10^{-11}$. The fitness values are shown graphically in Figure 2. The result of RK4 is obtained by implementing Matlab's built-in function Ode45. The solutions are graphically compared with RK4 given in Figure 6(a). Each variable $I(t), U(t)$, and $V(t)$ is drawn separately for 100 independent runs in Figures 6(c)-6(e). The obtained variable, known as weight, is also plotted in Figure 6(b). The weights are plotted with a $3 \mathrm{D}$ bar graph. The fitness value of the problem is evaluated through statistical operators minimum (MIN), maximum (MAX), and mean (MEAN). Data for statistical operators are given in Table 5.

The trained weights are used in equations (22)-(25) to get the solution for the CVRT model as follows:

$$
\begin{aligned}
& \widehat{I}(t)=\frac{-0.0000}{1+e^{-(0.1400 t-0.0905)}}+\cdots+\frac{-0.3924}{1+e^{-(1.4078 t-0.5155)}}, \\
& \widehat{U}(t)=\frac{0.4312}{1+e^{-(-3.8564 t-4.1435)}}+\cdots+\frac{2.1542}{1+e^{-(0.3071 t+0.5714)}}, \\
& \widehat{V}(t)=\frac{0.0916}{1+e^{-(-1.4469 t-1.1186)}}+\cdots+\frac{1.8651}{1+e^{-(1.0928 t+0.0706)}} \text {. }
\end{aligned}
$$

4.5. Problem 5. This problem is discussed for tumor growth ratio $(r)$ to rate of cell lysis $(\delta) f=0.36$, the inverse of burst size parameter $g=0.02$, and the viral decay $(\alpha)$ ratio to rate of cell lysis $(\delta) h=0.15$. These values updated the fitness function as

$$
E=\left(\begin{array}{c}
\frac{1}{10} \sum_{m=1}^{10}\left(\left(\frac{\mathrm{d} \widehat{I}_{m}}{\mathrm{~d} t}-0.36 \widehat{I}_{m}+\widehat{I}_{m} \widehat{V}_{m}\right)^{2}+\left(\frac{\mathrm{d} \widehat{U}_{m}}{\mathrm{~d} t}-\widehat{I}_{m} \widehat{V}_{m}+\widehat{U}_{m}\right)^{2}+\left(\frac{\mathrm{d} \widehat{V}_{m}}{\mathrm{~d} t}-\widehat{U}_{m}+0.02 \widehat{I}_{m} \widehat{V}_{m}+0.15 \widehat{V}_{m}\right)^{2}\right) \\
+\frac{1}{3}\left(\left(\widehat{I}_{0}-0.4\right)^{2}+\widehat{U}_{0}^{2}+\left(\widehat{V}_{0}-0.5\right)^{2}\right)
\end{array}\right)
$$

The associated condition are $I(0)=0.4, U(0)=0$, and $V(0)=0.5$.

The proposed scheme ANN-SQP-SCA is implemented to optimize the fitness function. The solution is compared with the solution of Range-Kutta order 4 (RK4). The set of weights for each variable, $I(t), U(t)$, and $V(t)$, are found with fitness $10^{-10}, 10^{-11}$, and $1.22 \times 10^{-10}$, respectively. The fitness values are shown graphically in Figure 2 . The result of 


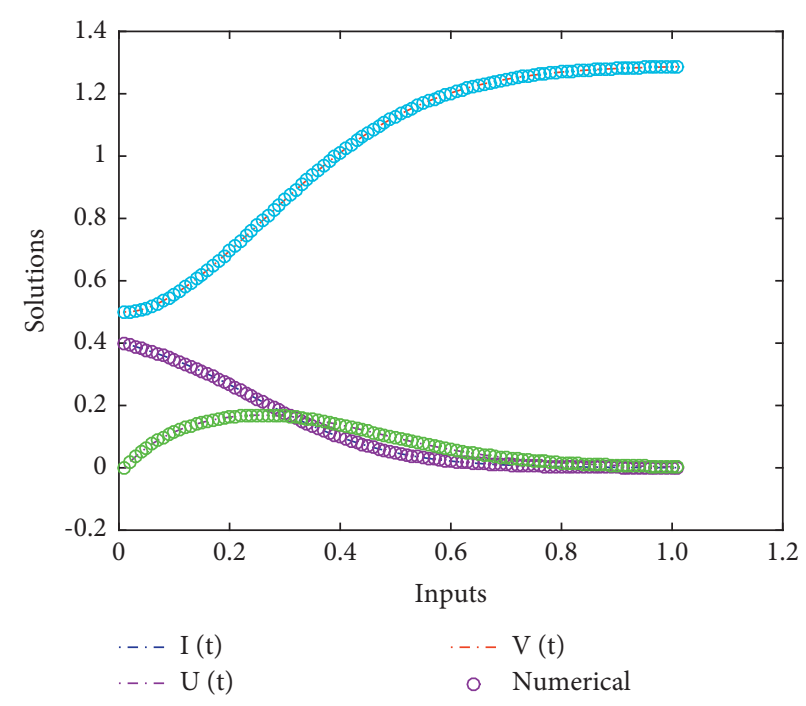

(a)

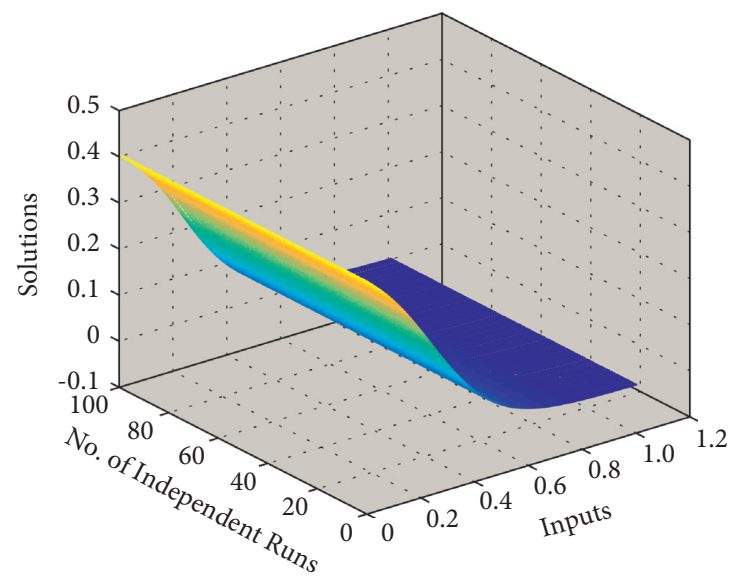

I ( $\mathrm{t})$

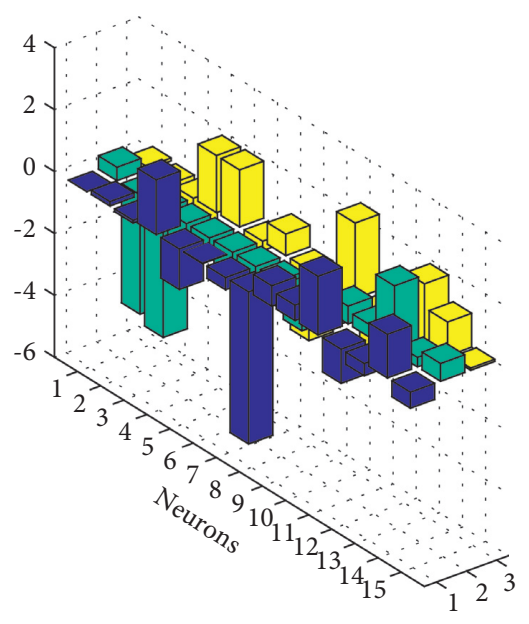

(b)

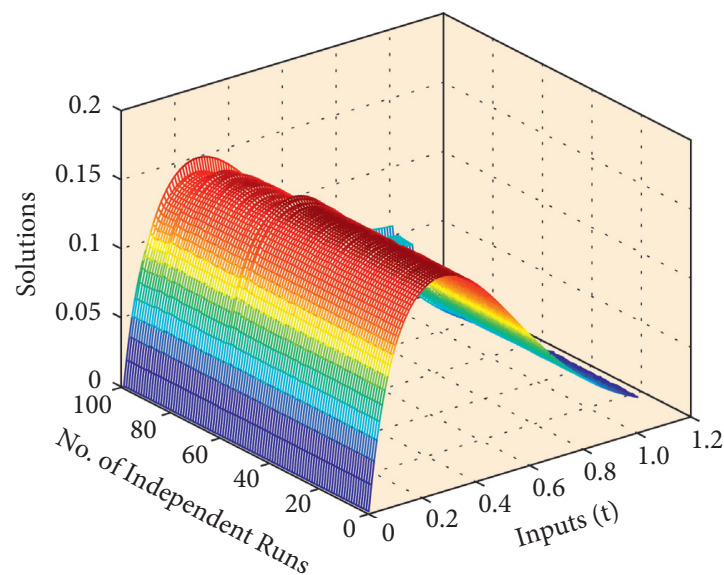

$\mathrm{U}(\mathrm{t})$

(c)

(d)

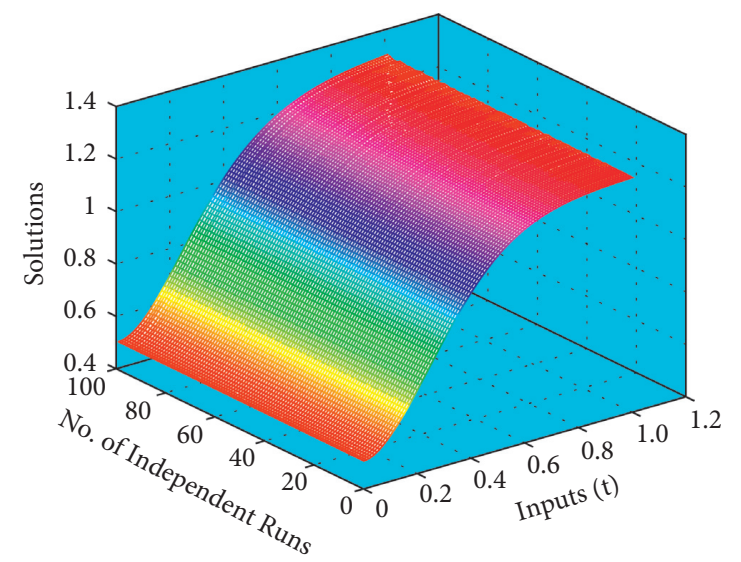

$\mathrm{V}(\mathrm{t})$

(e)

Figure 6: Problem 4: graphical evaluation for tumor growth ratio $(r)$ to rate of cell lysis $(\delta) f=0.36$, the inverse of burst size parameter $g=0.02$, and the viral decay $(\alpha)$ ratio to rate of cell lysis $(\delta) h=0$. (a) Solution $\mathrm{f}=0.36, \mathrm{~g}=0.02$, and $\mathrm{h}=0$ with initial conditions $I(0)=0.4, U$ $(0)=0$, and $V(0)=0.5$, (b) weights obtained by ANN-SCA-SQP for CVRT, (c) solutions of 100 independent runs of $I(t)$, (d) solutions of 100 independent runs of $U(t)$, and (e) solutions of 100 independent runs of $V(t)$. 


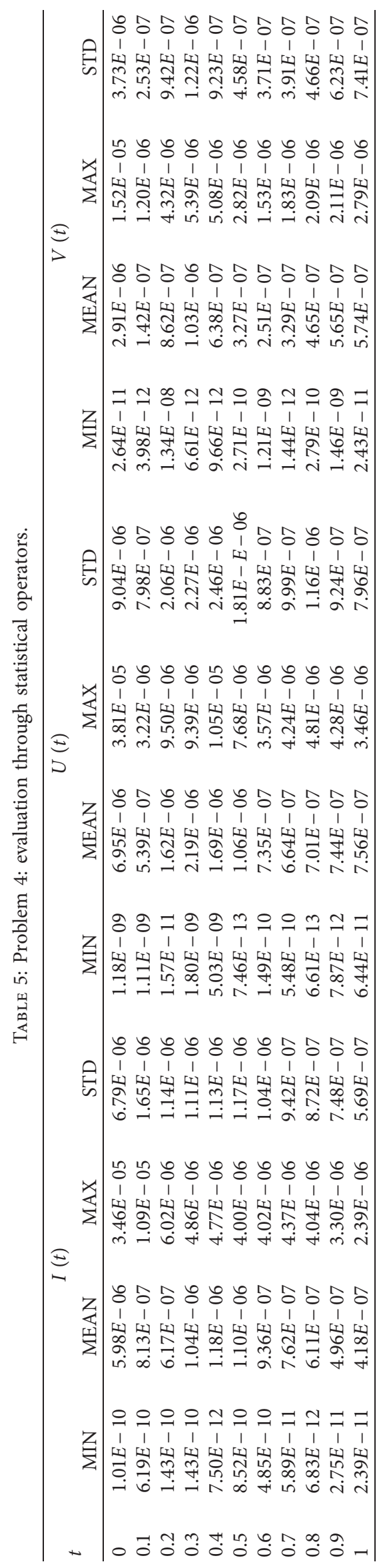


RK4 is obtained by implementing Matlab's built-in function Ode45. The solutions are graphically compared with RK4 given in Figure 7(a). Each variable $I(t), U(t)$, and $V(t)$ is drawn separately for 100 independent runs in Figures $7(\mathrm{c})-$ 7(e). The obtained variable, known as weight, is also plotted in Figure 7(b). The weights are plotted with a 3D bar graph. The fitness value of the problem is evaluated through statistical operators minimum (MIN), maximum (MAX), and mean (MEAN). Data for statistical operators are given in Table 6.

The trained weights are used in equations (22)-(25) to get the solution for the CVRT model as follows:

$$
\begin{aligned}
& \widehat{I}(t)=\frac{1.0000}{1+e^{-(-0.1002 t-0.4692)}}+\cdots+\frac{1.1039}{1+e^{-(1.5128 t+4.1814)}}, \\
& \widehat{U}(t)=\frac{1.6107}{1+e^{-(0.2301 t-0.3940)}}+\cdots+\frac{-0.9486}{1+e^{-(-1.4712 t-0.1529)}}, \\
& \widehat{V}(t)=\frac{-0.2229}{1+e^{-(-1.1342 t+0.3495)}}+\cdots+\frac{-1.5497}{1+e^{-(-1.7010 t-0.3150)}}
\end{aligned}
$$

The full form of the solution in equation (45) is given in Appendix with 14 decimal places to avoid any roundoff error. The overlapping of solutions with the results of RK4 shows the convergence of the proposed scheme. The solution is obtained for $t \in[0,1]$ with step size 0.01 , which gives 101 points. The small step size is taken to verify small variation in the solution.

This problem concerns the equilibrium point $(0,0, V)$. If $V=f$, then it will become $(0,0, f)$. Here, $V$ is arbitrary, but with the existence of viruses, the infected cells $I(t)$ decrease.

4.6. Evaluation of the Proposed Scheme by Performance Indices. To verify the reliability and consistency of proposed methodology, the proposed scheme is evaluated by performance indices, as defined in equations (29)-(32). The scheme is executed 100 times to collect large data for evaluation. The global optimizer SCA is executed in terms of ANN for generation of ANN weights. Then, the weights are provided to SQP as an initial point for further refinement. The calculated values of performance matrices are drawn in Figures 8 and 9 with line plots by taking $\log$ on the $Y$-axis for observing the small variation. For plotting, the data are arranged in descending order. It can be seen that the value of performance operator MAD lies between $10^{-03}-10^{-04}$; the same values are obtained for RMSE, and the values of ENSE are in between $10^{-06}$ and $10^{-12}$. The graph is drawn for each variable, $I(t), U(t)$, and $V(t)$, separately. The values of each variable are similar; that is, they lie in the same range which shows the consistency of the proposed scheme. Moreover, the scheme is evaluated by the global extension of performance operators named GMAD, GRMSE, and GENSE. The values for global operators are given in Table 7 . The values of GMAD lie in $10^{-04}-10^{-06}$, GRMSE between $10^{-04}-10^{-06}$, and the values of GENSE are in between $10^{-09}-10^{-16}$.
As the values of performance operators MAD, RMSE, and ENSE tend to zero, it verifies the consistency and reliability of the proposed methodology. The values of performance operators depend on the average value of number of runs. The fewer number of runs cannot verify the consistency of a technique. Therefore, the ANN-SCA-SQP algorithm is executed 100 times to collect a large dataset for better evaluation. The range of values for all the operators is impressive and shows the reliability of ANN-SCA-SQP algorithm. Furthermore, the convergence of the proposed algorithm is tested by using box plots. The obtained fitness values of each problem are drawn with the help of a line graph in Figure 2 and box plots in Figure 10. Each figure consists of three box plots for $I(t), U(t)$, and $V(t)$. The box plot shows five numerical values, for each variable, i.e., minimum, first quartile, median, third quartile, and maximum. This illustrates the fitness of solutions.

4.7. Complexity Analysis. For complexity analysis, the proposed scheme is executed 100 times to critically observe the convergence rate of the scheme. The found solutions through these executions are drawn graphically. As the solved problem is a 3 by 3 system of ODEs, solutions for each variable, i.e., $I(t), U(t)$, and $V(t)$, are drawn.

In problem 1, Figure 3(c) shows the result for $I(t)$, and it seems that the scheme is converged on each run and no worst result is shown. Similarly, the same phenomena can observed for the other variables, $U(t)$ and $V(t)$, in Figures 3(d) and 3(e). It concludes the best performance of the scheme.

For problem 2, the solutions for $I(t), U(t)$, and $V(t)$ are shown in Figures 4(c)-4(e), respectively, From the figures, it is observed that, for $I(t)$, no jumps are found meaning that the worst solutions are not computed by ANN-SCA-SQP algorithm. But, for $U(t)$ and $V(t)$, a few slightly worst solutions seem for 5-10 runs out of 100 . But, overall convergence is better. In problem 3, by observation Figures 5(c)-5(e) for each variable $I(t), U(t)$, and $V(t)$, out of 100 , about $10-15$ are such solutions which are slightly behind the numerical solution of RK4. In problem 4, all the solutions are smoothly converged to the numerical solution as can be observed from Figure 6(c)-6(e). Similarly, for problem 5, it can be seen from Figures 7(c)-7(e) that few solutions out of 100 are slightly behind numerical solutions.

The overall discussion concludes that the convergence rate of the scheme is high and the convergence of the scheme ensures. Also, the scheme is applicable for the solution of a large number of problems in comparison with state-of-theart techniques.

4.8. Tuning of Parameters. The ANN-SCA-SQP algorithm is evaluated by tuning its parameters, population size, and the number of neurons. The proposed algorithm is executed with 30 population size and 45 neurons. The results in term of absolute errors of each variable $I(t), U(t)$, and $V(t)$ are reported in Tables $8-10$. Tables 8 and 9 show the data for 


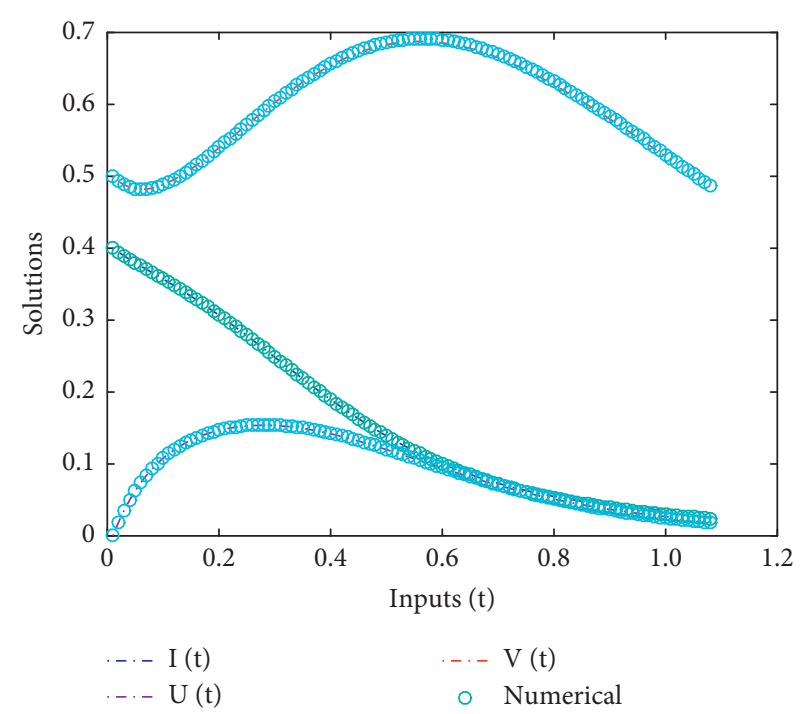

(a)

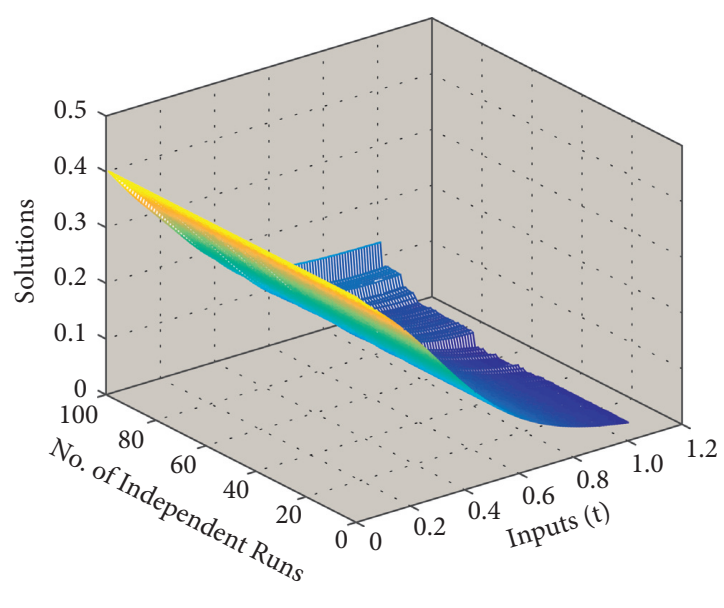

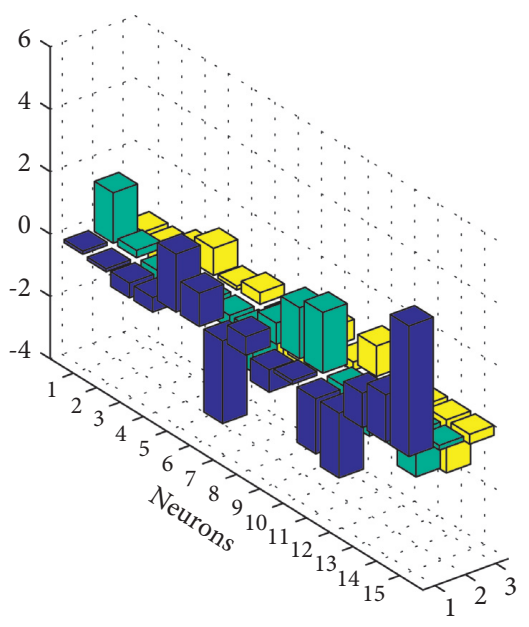

(b)

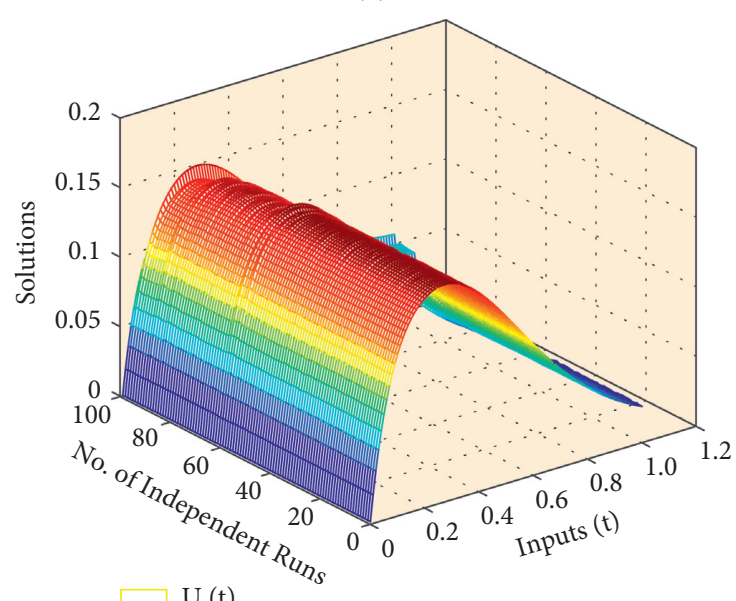

(d)

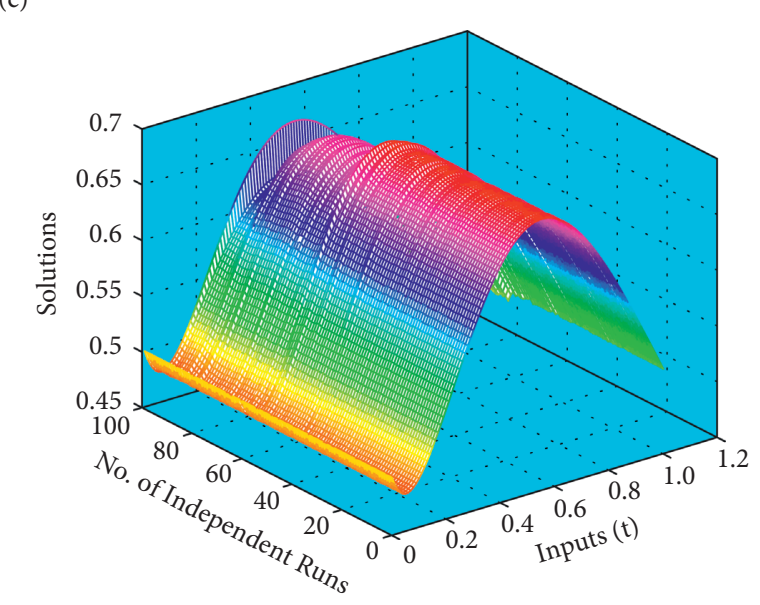

(e)

Figure 7: Problem 5: graphical evaluation for tumor growth ratio $(r)$ to rate of cell lysis $(\delta) f=0.36$, the inverse of burst size parameter $g=0.02$, and the viral decay $(\alpha)$ ratio to rate of cell lysis $(\delta) h=0.15$. (a) Solution $f=0.36, g=0.02$, and $h=0.15$ with initial conditions $I(0)=$ $0.4, U(0)=0$, and $V(0)=0.1$, (b) weights obtained by ANN-SCA-SQP for CVRT, (c) solutions of 100 independent runs of $I(t)$, (d) solutions of 100 independent runs of $U(t)$, and (e) solutions of 100 independent runs of $V(t)$. 


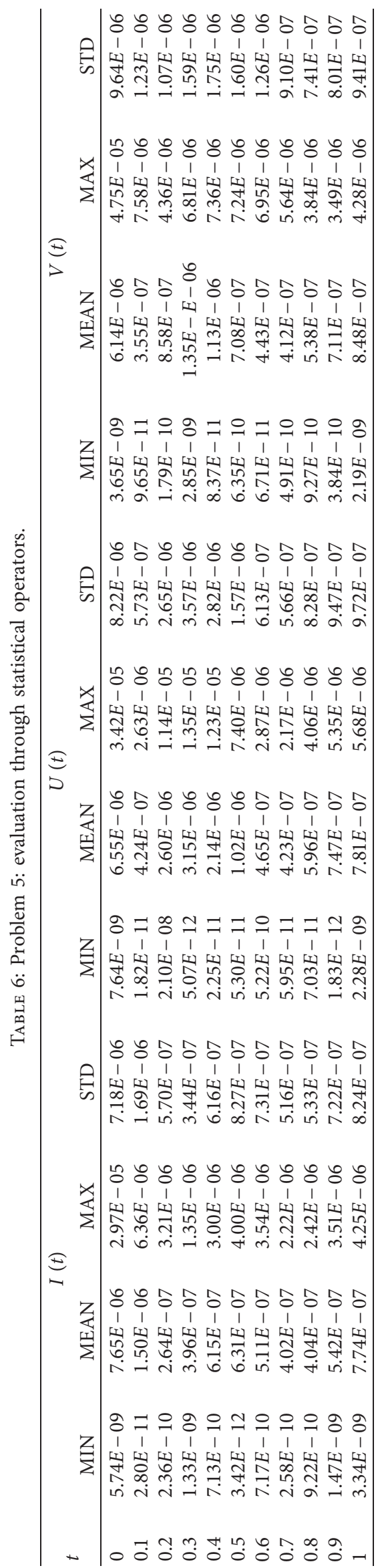




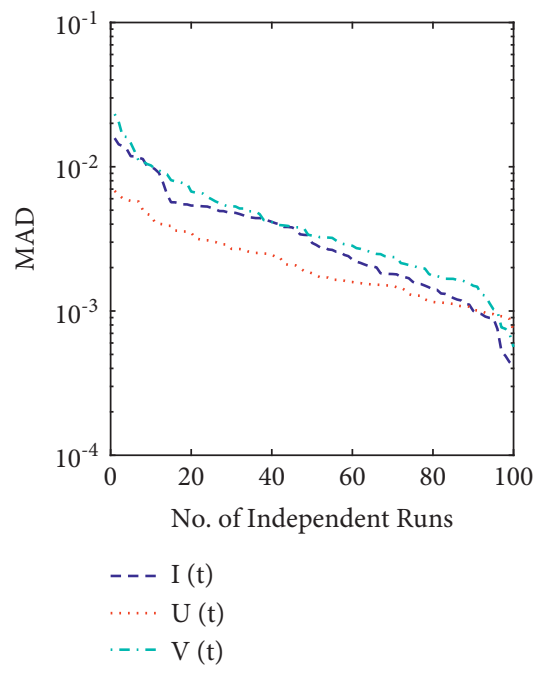

(a)

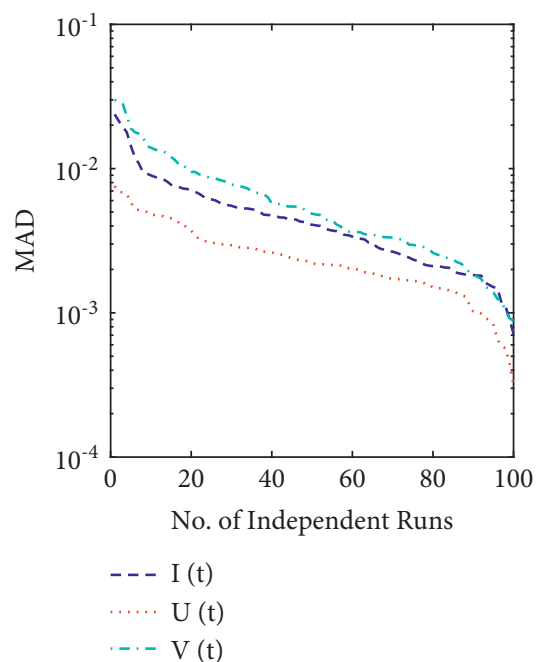

(d)

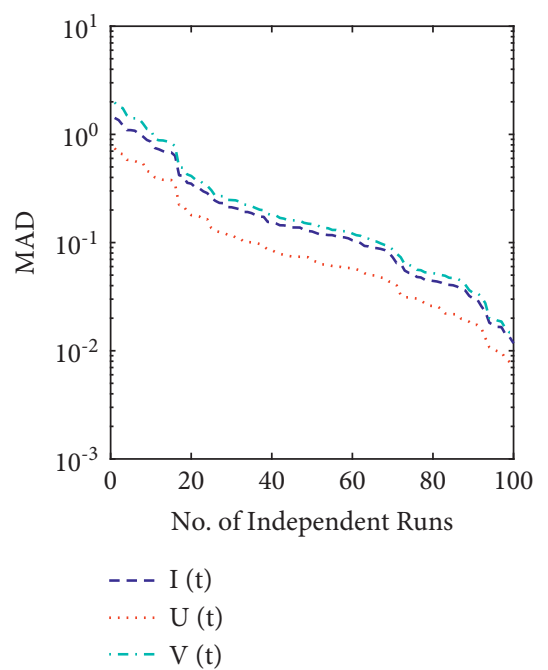

$(\mathrm{g})$

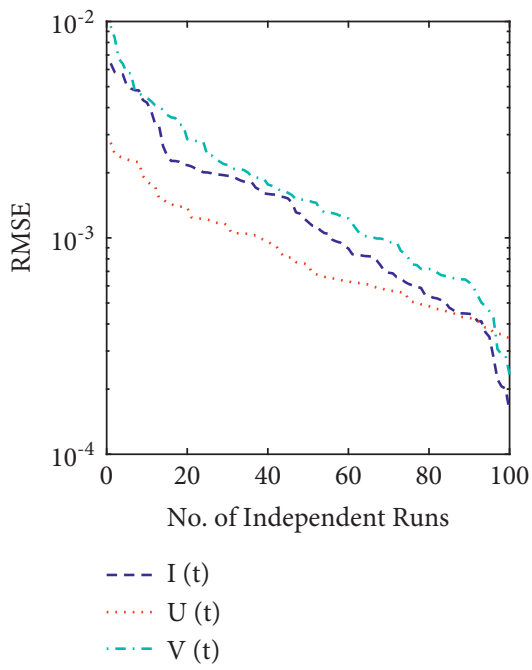

(b)

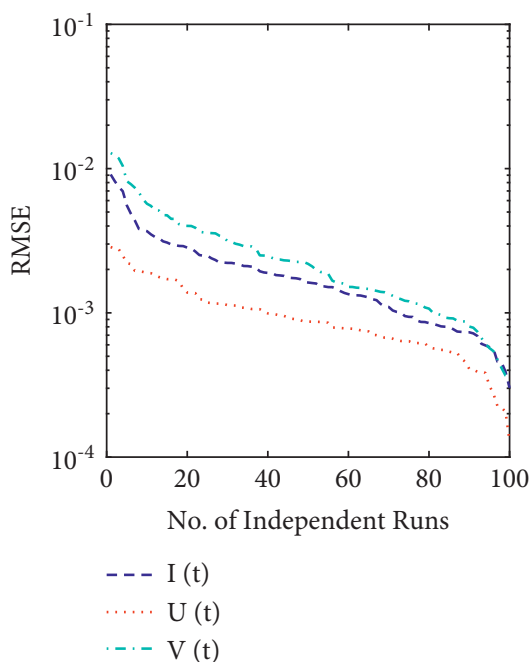

(e)

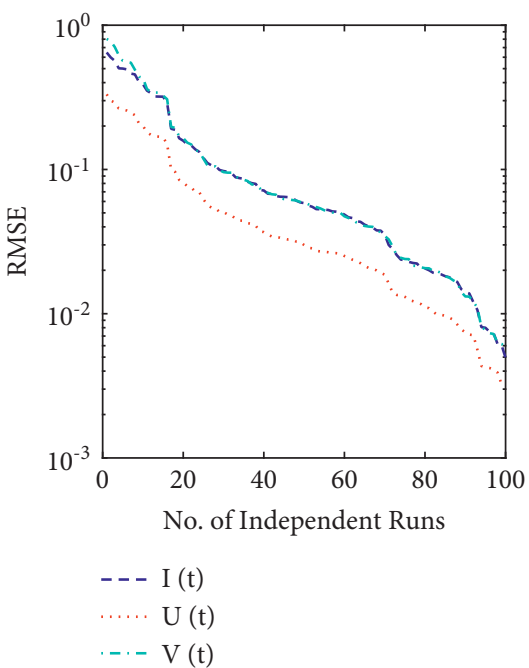

(h)

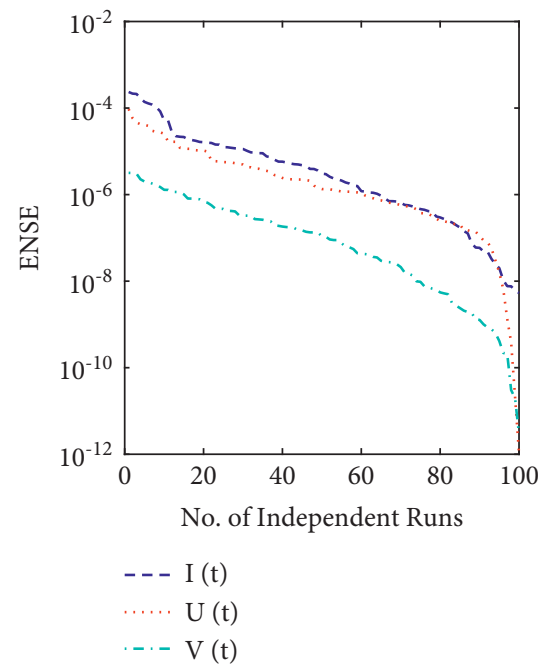

(c)

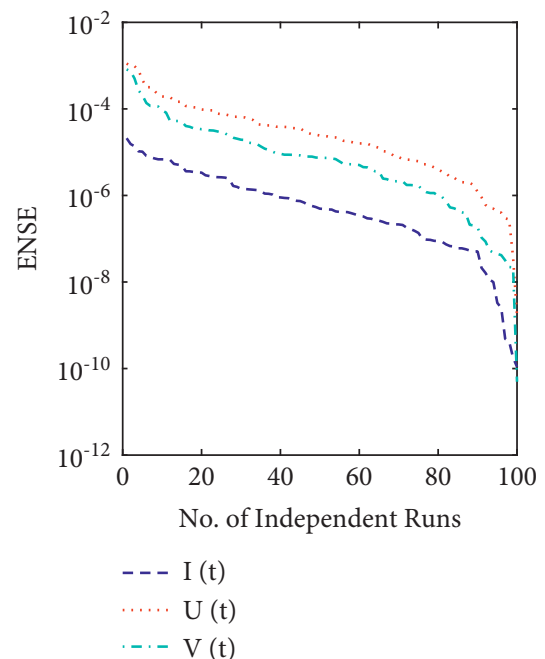

(f)

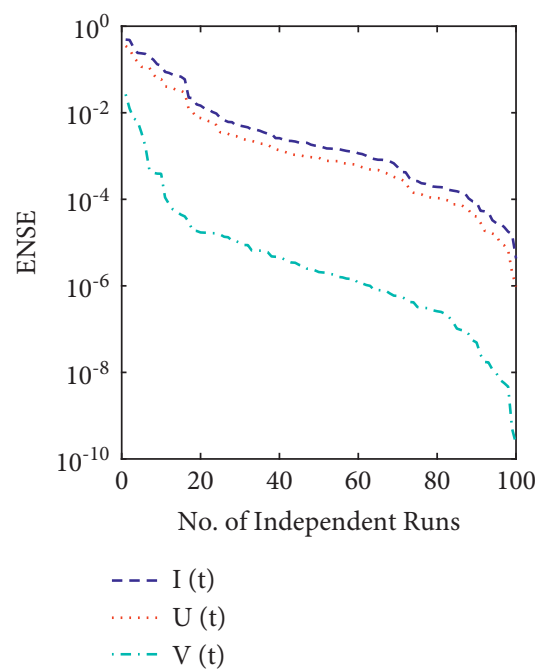

(i)

FIGURE 8: Graphs of performance operators. (a) MAD of problem 1, (b) RMSE of problem 1, (c) ENSE of problem 1, (d) MAD of problem 2, (e) RMSE of problem 2, (f) ENSE of problem 2, (g) MAD of problem 3, (h) RMSE of problem 3, and (i) ENSE of problem 3. 


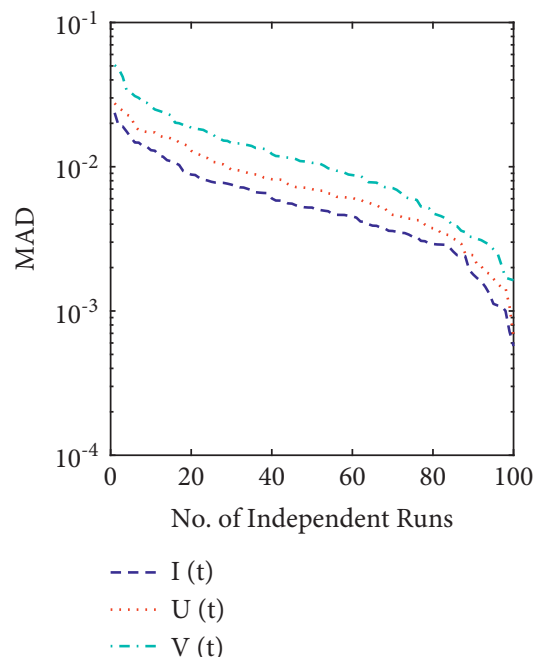

(a)

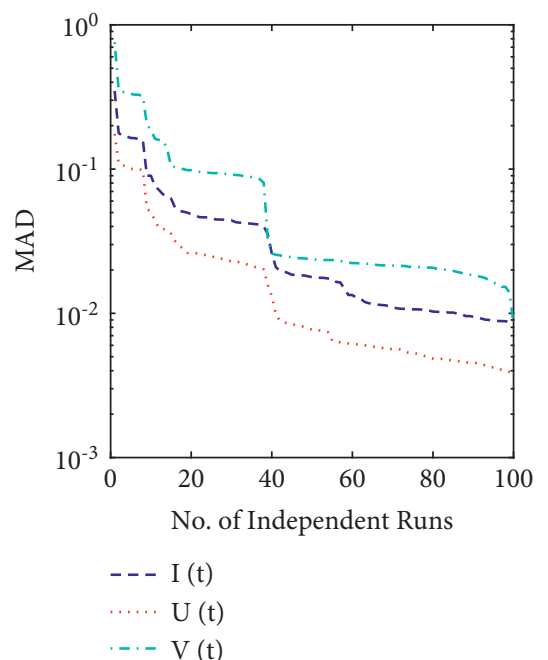

(d)

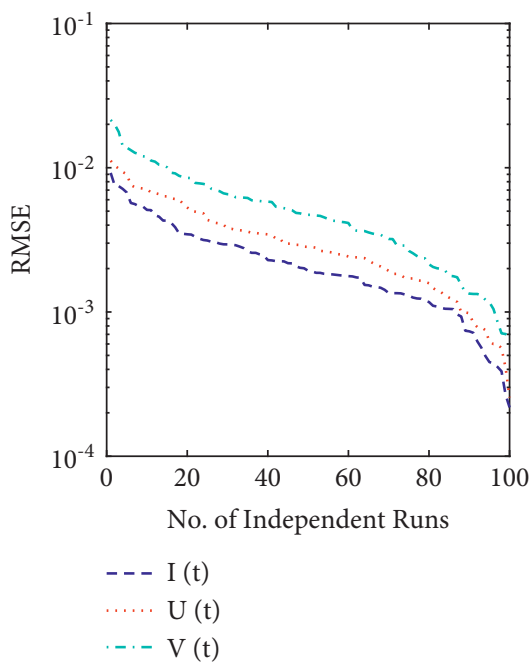

(b)

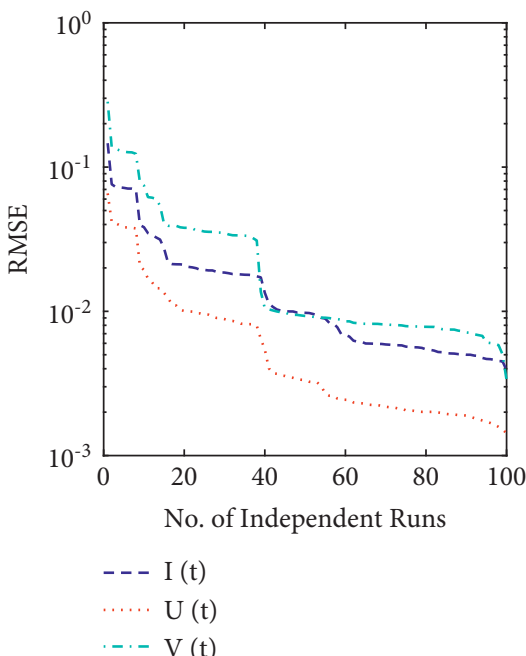

(e)

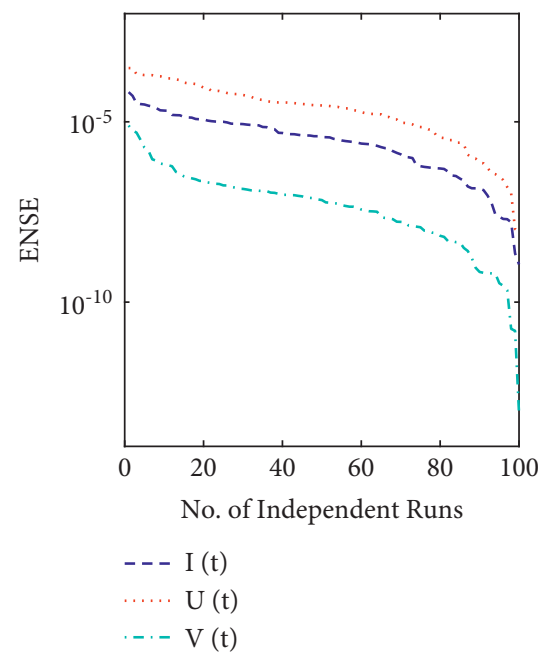

(c)

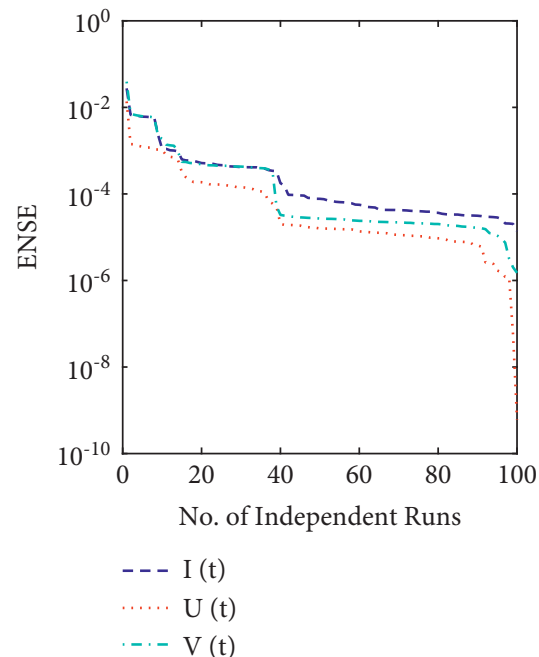

(f)

FIGURE 9: Graphs of performance operators. (a) MAD of problem 4, (b) RMSE of problem 4, (c) ENSE of problem 4, (d) MAD of problem 5, (e) RMSE of problem 5, and (f) ENSE of problem 5.

TABLE 7: Values of global performance operators.

\begin{tabular}{|c|c|c|c|c|}
\hline Problem & Operator & $I(t)$ & $U(t)$ & $V(t)$ \\
\hline 1 & $\begin{array}{l}\text { GMAD } \\
\text { GRMSE } \\
\text { GENSE }\end{array}$ & $\begin{array}{l}4.18 E-06 \\
1.58 E-06 \\
5.32 E-11\end{array}$ & $\begin{array}{l}7.31 E-06 \\
3.45 E-06 \\
1.04 E-14\end{array}$ & $\begin{array}{l}5.63 E-06 \\
2.33 E-06 \\
2.86 E-14 \\
\end{array}$ \\
\hline 2 & $\begin{array}{l}\text { GMAD } \\
\text { GRMSE } \\
\text { GENSE }\end{array}$ & $\begin{array}{l}7.16 E-04 \\
3.02 E-04 \\
1.09 E-10\end{array}$ & $\begin{array}{l}3.15 E-04 \\
1.28 E-04 \\
1.65 E-09\end{array}$ & $\begin{array}{l}8.83 E-04 \\
3.56 E-04 \\
4.96 E-11\end{array}$ \\
\hline 3 & $\begin{array}{l}\text { GMAD } \\
\text { GRMSE } \\
\text { GENSE }\end{array}$ & $\begin{array}{l}1.17 E-04 \\
4.94 E-05 \\
4.29 E-08\end{array}$ & $\begin{array}{l}7.28 E-05 \\
3.23 E-05 \\
9.64 E-09\end{array}$ & $\begin{array}{l}1.47 E-04 \\
5.80 E-05 \\
2.23 E-12 \\
\end{array}$ \\
\hline 4 & $\begin{array}{l}\text { GMAD } \\
\text { GRMSE } \\
\text { GENSE }\end{array}$ & $\begin{array}{l}5.71 E-06 \\
2.18 E-06 \\
1.11 E-11\end{array}$ & $\begin{array}{l}6.64 E-06 \\
2.56 E-06 \\
8.85 E-11\end{array}$ & $\begin{array}{l}1.63 E-05 \\
6.78 E-06 \\
8.23 E-16\end{array}$ \\
\hline 5 & $\begin{array}{l}\text { GMAD } \\
\text { GRMSE } \\
\text { GENSE }\end{array}$ & $\begin{array}{l}8.64 E-05 \\
3.89 E-05 \\
1.38 E-07\end{array}$ & $\begin{array}{l}3.70 E-05 \\
1.43 E-05 \\
6.03 E-12\end{array}$ & $\begin{array}{l}8.40 E-05 \\
3.34 E-05 \\
1.50 E-08\end{array}$ \\
\hline
\end{tabular}




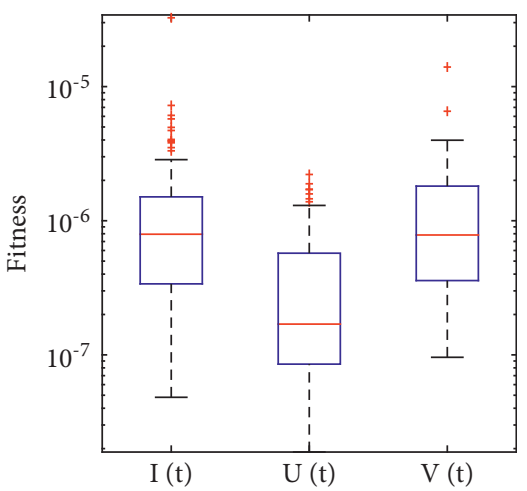

(a)

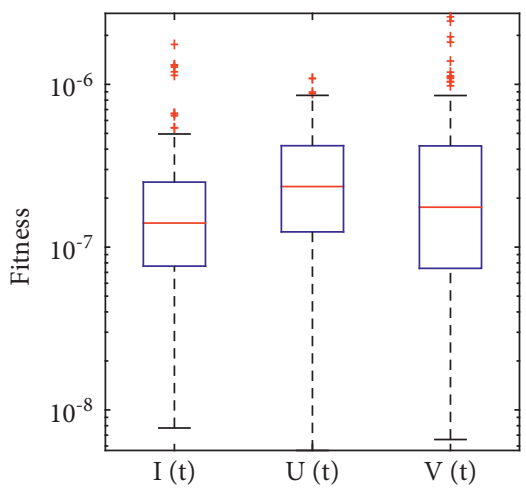

(b)

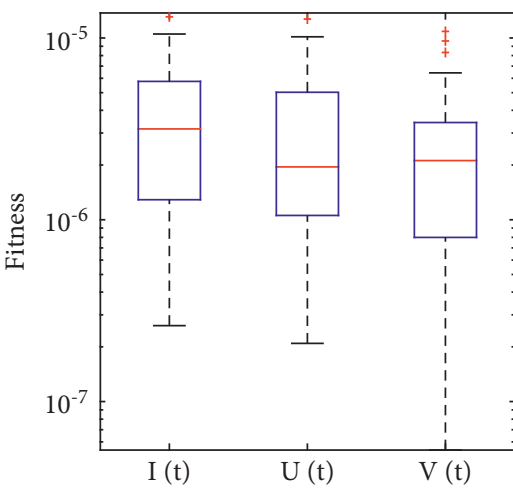

(c)

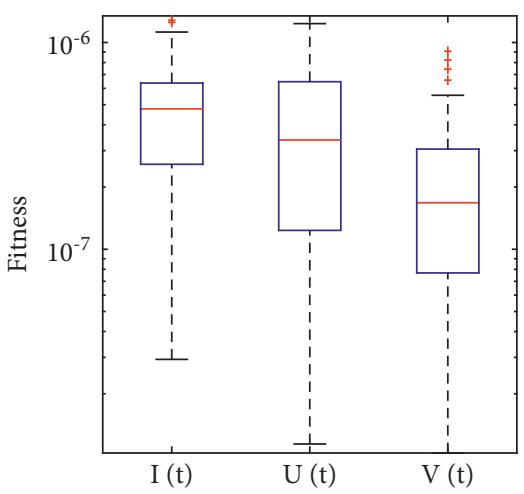

(d)

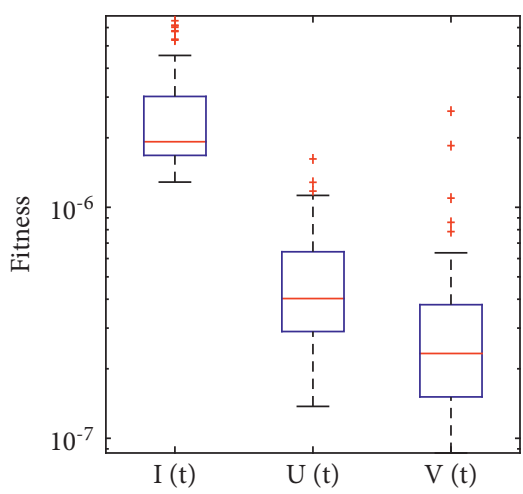

(e)

Figure 10: Fitness evaluation through box plots. (a) Fitness of problem 1, (b) fitness of problem 2, (c) fitness of problem 3, (d) fitness of problem 4, and (e) fitness of problem 5.

Table 8: Problem 1: analysis based on the variation of population size.

\begin{tabular}{|c|c|c|c|c|c|c|c|c|c|}
\hline \multirow{2}{*}{$\begin{array}{l}\text { Variable } \\
\text { Input }(\mathrm{t}) \backslash \text { population }\end{array}$} & \multicolumn{3}{|c|}{$I(t)$} & \multicolumn{3}{|c|}{$U(t)$} & \multicolumn{3}{|c|}{$V(t)$} \\
\hline & 20 & 30 & 40 & 20 & 30 & 40 & 20 & 30 & 40 \\
\hline 0 & $1.54 E-06$ & $3.77 E-12$ & $1.26 E-05$ & $1.54 E-06$ & $1.35 E-09$ & $1.26 E-05$ & $2.47 E-06$ & $3.07 E-07$ & $6.67 E-06$ \\
\hline 0.1 & $2.99 E-05$ & $1.17 E-10$ & $2.82 E-05$ & $2.99 E-05$ & $1.94 E-11$ & $2.82 E-05$ & $2.96 E-05$ & $6.13 E-08$ & $1.17 E-05$ \\
\hline 0.2 & $7.48 E-05$ & $1.38 E-10$ & 0.000132 & $7.48 E-05$ & $2.80 E-10$ & $1.32 E-04$ & $7.36 E-05$ & $1.83 E-07$ & $1.82 E-05$ \\
\hline 0.3 & 0.00019 & $2.42 E-09$ & 0.000313 & 0.00019 & $4.10 E-10$ & 0.000313 & $7.28 E-05$ & $8.43 E-08$ & $3.96 E-05$ \\
\hline 0.4 & 0.00026 & $2.28 E-12$ & 0.000416 & $2.60 E-04$ & $8.88 E-11$ & 0.000416 & $3.38 E-05$ & $1.53 E-08$ & $6.47 E-05$ \\
\hline 0.5 & 0.00027 & $2.18 E-11$ & 0.000408 & $2.70 E-04$ & $8.07 E-11$ & 0.000408 & $2.59 E-05$ & $5.49 E-08$ & $8.96 E-05$ \\
\hline 0.6 & 0.000227 & $4.00 E-10$ & 0.000308 & $2.27 E-04$ & $6.59 E-11$ & 0.000308 & $8.43 E-05$ & $4.21 E-08$ & $1.10 E-04$ \\
\hline 0.7 & 0.000157 & $2.69 E-12$ & 0.00017 & 0.000157 & $5.84 E-11$ & 0.00017 & $1.11 E-04$ & $4.76 E-08$ & $1.10 E-04$ \\
\hline 0.8 & $7.82 E-05$ & $3.77 E-12$ & $4.19 E-05$ & $7.82 E-05$ & $4.88 E-11$ & $4.19 E-05$ & $9.70 E-05$ & $1.61 E-07$ & $8.80 E-05$ \\
\hline 0.9 & $2.71 E-06$ & $9.49 E-10$ & $4.32 E-E-05$ & $2.71 E-06$ & $8.83 E-11$ & $4.32 E-05$ & $5.28 E-05$ & $6.42 E-08$ & $5.73 E-05$ \\
\hline 1 & $6.38 E-05$ & $5.16 E-11$ & $7.24 E-05$ & $6.38 E-05$ & $1.52 E-13$ & $7.24 E-05$ & $8.01 E-07$ & $8.51 E-09$ & $3.58 E-05$ \\
\hline
\end{tabular}

variation of population size, and Table 10 presents data for variation of neurons.

The variation of population is tested on problem 1 and problem 5. From Tables 8 and 9, it can be observed that, for 20 population size, the absolute errors are between $10^{-04}$ and $10^{-06}$, the errors for 30 population size lie between $10^{-09}$ and $10^{-12}$, and the errors for 40 population size are in between $10^{-03}$ and $10^{-06}$. It can be clearly observed that the performance of ANN-SCA-SQP algorithm on 30 population is much better than the rest of the population.

Similarly, the performance of ANN-SCA-SQP algorithm is evaluated on the basis of variation of neurons. For this, the proposed technique is executed for problem 3 only. Table 10 reports the errors for 27, 45, and 90 neurons. From the table, it is clearly observed that the technique outperformed 45 neurons because the error in the model for 45 neurons is much less than the rest of number of neurons. 
TABLE 9: Problem 5: analysis based on the variation of population size.

\begin{tabular}{|c|c|c|c|c|c|c|c|c|c|}
\hline \multirow{2}{*}{$\begin{array}{l}\text { Variable } \\
\text { Input }(\mathrm{t}) \backslash \text { population }\end{array}$} & \multicolumn{3}{|c|}{$I(t)$} & \multicolumn{3}{|c|}{$U(t)$} & \multicolumn{3}{|c|}{$V(t)$} \\
\hline & 20 & 30 & 40 & 20 & 30 & 40 & 20 & 30 & 40 \\
\hline 0 & $5.02 E-05$ & $1.01 E-10$ & $1.28 E-03$ & $5.92 E-05$ & $1.18 E-09$ & $2.90 E-04$ & $1.89 E-05$ & $2.64 E-11$ & $1.74 E-04$ \\
\hline 0.1 & $1.07 E-05$ & $6.19 E-10$ & $2.80 E-03$ & $2.33 E-04$ & $1.11 E-09$ & $2.96 E-04$ & $1.50 E-04$ & $3.98 E-12$ & $2.29 E-04$ \\
\hline 0.2 & 0.000104 & $1.43 E-10$ & 0.004184 & 0.00014 & $1.57 E-11$ & 0.000293 & $5.21 E-05$ & $1.34 E-08$ & $2.35 E-04$ \\
\hline 0.3 & 0.000203 & $1.43 E-10$ & 0.005459 & 0.000115 & $1.80 E-09$ & 0.000252 & 0.000109 & $6.61 E-12$ & 0.00025 \\
\hline 0.4 & 0.000257 & $7.50 E-12$ & 0.006644 & 0.000396 & $5.03 E-09$ & 0.000171 & 0.000242 & $9.66 E-12$ & 0.000296 \\
\hline 0.5 & 0.00026 & $8.52 E-10$ & 0.007765 & 0.000612 & $7.46 E-13$ & $6.01 E-05$ & $3.05 E-04$ & $2.71 E-10$ & $3.86 E-04$ \\
\hline 0.6 & 0.000225 & $4.85 E-10$ & 0.008851 & 0.000717 & $1.49 E-10$ & $5.71 E-05$ & $2.81 E-04$ & $1.21 E-09$ & $5.28 E-04$ \\
\hline 0.7 & 0.00017 & $5.89 E-11$ & 0.009934 & 0.000695 & $5.48 E-10$ & 0.000154 & 0.000178 & $1.44 E-12$ & 0.000726 \\
\hline 0.8 & 0.000111 & $6.83 E-12$ & 0.011046 & 0.00057 & $6.61 E-13$ & 0.000202 & $2.34 E-05$ & $2.79 E-10$ & $9.69 E-04$ \\
\hline 0.9 & $6.11 E-05$ & $2.75 E-11$ & $1.22 E-02$ & $3.83 E-04$ & $7.87 E-12$ & $1.74 E-04$ & $1.49 E-04$ & $1.46 E-09$ & $1.24 E-03$ \\
\hline 1 & $2.39 E-05$ & $2.39 E-11$ & $1.35 E-02$ & $1.69 E-04$ & $6.44 E-11$ & $5.59 E-05$ & $3.12 E-04$ & $2.43 E-11$ & $1.54 E-03$ \\
\hline
\end{tabular}

TABLe 10: Problem 3: analysis based on the variation of the number of neurons.

\begin{tabular}{|c|c|c|c|c|c|c|c|c|c|}
\hline \multirow{2}{*}{$\begin{array}{l}\text { Variable } \\
\text { Input }(\mathrm{t}) \backslash \text { neurons }\end{array}$} & \multicolumn{3}{|c|}{$I(t)$} & \multicolumn{3}{|c|}{$U(t)$} & \multicolumn{3}{|c|}{$V(t)$} \\
\hline & 27 & 45 & 90 & 27 & 45 & 90 & 27 & 45 & 90 \\
\hline 0 & 0.000172 & $1.06 E-09$ & $2.18 E-05$ & 0.000172 & $8.12 E-12$ & 0.000125 & 0.001216 & $8.06 E-10$ & $5.50 E-05$ \\
\hline 0.1 & 0.000312 & $7.40 E-10$ & 0.000361 & 0.000312 & $1.92 E-08$ & 0.0001 & 0.002978 & $5.13 E-09$ & 0.000204 \\
\hline 0.2 & 0.00073 & $7.90 E-11$ & 0.000519 & 0.00073 & $4.04 E-10$ & 0.000211 & 0.004477 & $4.29 E-09$ & 0.00027 \\
\hline 0.3 & 0.001279 & $8.48 E-11$ & 0.000507 & 0.001279 & $5.15 E-09$ & 0.000239 & 0.005745 & $5.58 E-12$ & 0.000277 \\
\hline 0.4 & 0.00187 & $3.82 E-09$ & 0.000375 & 0.00187 & $1.47 E-09$ & 0.00021 & 0.00681 & $6.30 E-10$ & 0.000244 \\
\hline 0.5 & 0.002463 & $1.10 E-09$ & 0.000166 & 0.002463 & $9.94 E-11$ & 0.000145 & 0.007697 & $4.60 E-11$ & 0.000187 \\
\hline 0.6 & 0.003044 & $1.08 E-11$ & $8.28 E-05$ & 0.003044 & $3.60 E-10$ & $6.02 E-05$ & 0.008431 & $4.16 E-10$ & 0.00012 \\
\hline 0.7 & 0.003607 & $4.46 E-12$ & 0.00034 & 0.003607 & $7.72 E-15$ & $2.80 E-05$ & 0.009035 & $2.70 E-10$ & $5.55 E-05$ \\
\hline 0.8 & 0.004154 & $2.73 E-10$ & 0.000583 & 0.004154 & $2.71 E-09$ & 0.00011 & 0.009526 & $2.90 E-10$ & $7.59 E-07$ \\
\hline 0.9 & 0.004689 & $5.12 E-10$ & 0.000793 & 0.004689 & $3.08 E-09$ & 0.000183 & 0.009922 & $4.65 E-12$ & $3.95 E-05$ \\
\hline 1 & 0.005218 & $1.18 E-10$ & 0.000958 & 0.005218 & $1.08 E-08$ & 0.000242 & 0.010237 & $1.49 E-09$ & $6.19 E-05$ \\
\hline
\end{tabular}

\section{Conclusions}

The oncolytic viruses are considered an effective treatment of cancer. But, the effectiveness of oncolytic viruses is limited, especially in aggressive tumors. In this work, the model is proposed to enhance the treatment of cancer through virotherapy with aggressive tumors. The model dynamics are evaluated in many parameters such as the absence of tumor growth and viral decay given in ratios of tumor growth $(r)$ to rate of cell lysis and the viral decay $(\alpha)$ ratio to rate of cell lysis, respectively. For simplicity, the parameters of the system are transformed and derived from system (4). For the solution of system (4), a hybridized technique of SCA and SQP is proposed with the worth of ANN. For a solution of system (4), the model is transformed to an ANN form and a fitness function is constructed using mean square error. A large dataset is calculated for multiple runs for the evaluation of the ANN-SCA-SQP algorithm. The scheme is evaluated by performance operators and its global extensions. Moreover, the fitness is evaluated graphically such as box plot. Through evaluation, it is found that the scheme is consistent, robust, and effective. The same scheme can be modified by implementing different activation functions. The methodology can be further utilized solving biomathematics, engineering, and physical problems.

\section{Abbreviations \\ ANN: Artificial neural network \\ SCA: Sine-cosine algorithm \\ SQP: Sequential quadratic programming \\ CVRT: Cancer virotherapy \\ MAD: Mean absolute deviation \\ RMSE: Root mean squared error \\ ENSE: Error in Nash-Sutcliffe efficiency.}

\section{Appendix}

Full forms of all the solutions are given in this section. Solution of problem 1: 


$$
\begin{aligned}
& \widehat{I}(t)=\frac{-0.01908663071991}{1+e^{-(-1.7427587553733 t+0.43880676467695)}}+\frac{0.57636851069413}{1+e^{-(-2.05483312206432 t-1.51279831940580)}} \\
& +\frac{0.11348110707162}{1+e^{-(-0.26680586948821 t-0.95880080821399)}}+\frac{1.02716171085393}{1+e^{-(-0.75107277522778 t+0.02119782447704)}} \\
& +\frac{-0.12265909533986}{1+e^{-(-2.3878459478257 t-0.61954169304901)}} \\
& \widehat{U}(t)=\frac{-1.06738510783545}{1+e^{-(-2.4095986340855 t-2.36669544479462)}}+\frac{0.00005698831622}{1+e^{-(0.01707353586923 t+0.00516606039046)}} \\
& +\frac{1.44274124201514}{1+e^{-(-0.61114780109026 t+0.55443859010272)}}+\frac{-1.58315352377259}{1+e^{-(-0.60000125315628 t-0.53538611019809)}} \\
& +\frac{-0.54498890438087}{1+e^{-(-0.60046414945646 t-0.23752663059692)}} \\
& \widehat{V}(t)=\frac{0.83230461358761}{1+e^{-(-0.21553261740414 t+0.32088763825645)}}+\frac{-1.15725352490915}{1+e^{-(-0.6601729156889 t+0.60367078501160)}} \\
& +\frac{0.83190784385561}{1+e^{-(-0.13937605750615 t+0.15897785716152)}}+\frac{0.52678493269401}{1+e^{-(-0.32518905233754 t-0.10854076304947)}} \\
& 0.7149907129149 \\
& +\frac{0.7149007129149}{1+e^{-(-2.31745054344404 t-2.25697503050407)}} \text {. }
\end{aligned}
$$

Solution of problem 2:

$$
\begin{aligned}
\widehat{I}(t)= & \frac{-0.00599366116434}{1+e^{-(-1.84937629052043 t+0.5099928234099)}}+\frac{0.29870864258774}{1+e^{-(0.43331546998478 t-0.46419231250538)}} \\
& +\frac{0.01374287509385}{1+e^{-(-2.22357196646945 t-0.53031108198807)}} \frac{0.70984937925103}{1+e^{-(-0.32334072478998 t+0.4383595914203)}} \\
& +\frac{-0.28141365492929}{1+e^{-(0.15686110604005 t+0.10576235234503)}}, \\
\widehat{U}(t)= & \frac{0.46576409855291}{1+e^{-(-0.25778414421679 t-0.01232021800099)}}+\frac{-0.11973901454464}{1+e^{-(-0.0558784821408 t+0.05072281204498)}} \\
& +\frac{-0.13271601599839}{1+e^{-(0.05320845584616 t+0.09704140793661)}}+\frac{-0.41736785455915}{1+e^{-(-0.33972792127982 t+0.01773769436056)}} \\
& +\frac{0.13203992298900}{1+e^{-(1.86201909140970 t+1.61046234132630)}}, \\
\widehat{V}(t)= & \frac{1.06397800332594}{1+e^{-(-0.14096838366040 t+0.28136014150827)}}+\frac{-1.10908696525454}{1+e^{-(-0.25673459511011 t+0.25692882213688)}} \\
& +\frac{0.23783569093672}{1+e^{-(-0.39293380250354 t-0.27200551063120)}}+\frac{0.07011107915698}{1+e^{-(-2.03980243421242 t-1.22616152138698)}} \\
& +\frac{0.22930304523515 t+0.03417748600235)}{1+}
\end{aligned}
$$

Solution of problem 3: 


$$
\begin{aligned}
\widehat{I}(t)= & \frac{0.00005064671963}{1+e^{-(0.91739425391987 t+0.20151282655898)}}+\frac{2.79676131118331}{1+e^{-(-0.58716175066135 t+3.08097561264812)}} \\
& +\frac{3.89798395539748}{1+e^{-(-0.93857598420893 t+5.45455853699118)}}+\frac{-2.35177680987183}{1+e^{-(-0.50105792267907 t+1.70417416852641)}} \\
& +\frac{-4.20850681202890}{1+e^{-(-0.78666971825920 t+4.57594710022521)}}, \\
\widehat{U}(t)= & \frac{0.29465880974700}{1+e^{-(-0.46205354373198 t-0.03717537716431)}}+\frac{-0.28100336217337}{1+e^{-(-0.63651781531408 t-0.09419821618922)}} \\
& +\frac{-3.44926321894795}{1+e^{-(-0.87499797267719 t+5.84323751817002)}}+\frac{-0.02533517624382}{1+e^{-(-2.80345714283232 t-0.26839438345355)}} \\
& +\frac{-3.45987601105871}{1+e^{-(-0.83183083661539 t+5.10453738628266)}}, \\
\widehat{V}(t)= & \frac{-1.14533668686484}{1+e^{-(-0.57409231224049 t+2.42523658199856)}}+\frac{0.26341954223219}{1+e^{-(0.36109769125255 t-3.75115778978136)}} \\
& +\frac{3.36966873878923}{1+e^{-(-0.29332641533667 t+0.18023795880885)}}+\frac{1.72348033989928}{1+e^{-(0.87636550576763 t-5.51906035772170)}} \\
& +\frac{-1.25503997625527}{1+(-0.60474451884446 t+0.22203891361175)} .
\end{aligned}
$$

Solution of problem 4:

$$
\begin{aligned}
& \widehat{I}(t)=\frac{-0.00000391767700}{1+e^{-(0.43115288195420 t+0.09163208113352)}}+\frac{0.14004409904071}{1+e^{-(-3.85644023499471 t-1.44692152222607)}} \\
& +\frac{-0.09050817566168}{1+e^{-(-4.14350709071546 t-1.11859678583174)}}+\frac{1.76864504058779}{1+e^{-(-0.73019391914329 t+1.84447982846083)}} \\
& -1.31074125251591 \\
& +\frac{-1.31074125251591}{1+e^{-(-0.70597808230969 t+1.84300062930665)}}, \\
& \widehat{U}(t)=\frac{-0.01290829995855}{1+e^{-(-1.08634059052199 t-0.30271510287789)}}+\frac{-0.39298288557352}{1+e^{-(-0.51127509262411 t+0.69613204466806)}} \\
& +\frac{-4.91583487264401}{1+e^{-(-0.86869421466160 t-2.28617515420093)}}+\frac{0.66123356250200}{1+e^{-(-1.14912984669606 t-0.40490975740441)}} \\
& +\frac{0.49651982078025}{1+e^{-(-0.65549713563820 t+2.44121814992890)}} \\
& \widehat{V}(t)=\frac{1.89212889740980}{1+e^{-(0.57221147821301 t-1.27442482230087)}}+\frac{-1.10136760888200}{1+e^{-(0.40426872098468 t-0.71783640953013)}} \\
& +\frac{-0.39237666404926}{1+e^{-(2.15422959030667 t+1.86510320642869)}}+\frac{1.40777398462851}{1+e^{-(0.30714424357335 t+1.09277874606487)}} \\
& -0.51552118752434 \\
& +\frac{-0.51552118752434}{1+e^{-(0.57138735375854 t+0.07062208745045)}} .
\end{aligned}
$$

Solution of problem 5: 


$$
\begin{aligned}
& \widehat{I}(t)=\frac{-0.00859463071392}{1+e^{-(-0.87024115209508 t+0.39460896786322)}}+\frac{1.13640297774529}{1+e^{-(-0.59905049527660 t+0.17088883788221)}} \\
& +\frac{0.33741608774768}{1+e^{-(-0.18960399251998 t-0.79411661042353)}}+\frac{-0.78464197267168}{1+e^{-(-0.83853480431552 t+0.42518124831745)}} \\
& 0.29534056262380 \\
& +\overline{1+e^{-(-0.41116980035838 t+0.13986062121403)}} \\
& \widehat{U}(t)=\frac{-0.12118520694302}{1+e^{-(0.29146780078467 t+0.64950809421930)}}+\frac{0.73349234278055}{1+e^{-(0.66394205424639 t-0.21318282907264)}} \\
& +\frac{-1.67135056809850}{1+e^{-(0.41138887024662 t+0.04878744456714)}}+\frac{0.51102395623546}{1+e^{-(2.12697609049951 t+1.31616368533260)}} \\
& 0.54806198119498 \\
& +\frac{e^{-(0.58145219794766 t-0.51570357305880)}}{1+} \\
& \widehat{V}(t)=\frac{-0.64200372200432}{1+e^{-(0.61755947255081 t+0.11456773690377)}}+\frac{0.64463738623510}{1+e^{-(0.44484405350485 t-0.51037698474455)}} \\
& +\frac{-1.18609854644186}{1+e^{-(-0.49027595136870 t+0.68477500730206)}}+\frac{1.90430292536142}{1+e^{-(-2.05047903280200 t-3.57238361188037)}} \\
& 2.01506128595190 \\
& +\frac{e^{-(-0.16491698824466 t+0.67240990771832)}}{1+}
\end{aligned}
$$

\section{Data Availability}

The data used to support the findings of this study are available from the corresponding author upon request.

\section{Conflicts of Interest}

The authors declare no conflicts of interest regarding this work.

\section{References}

[1] H. R. Bierman, D. M. Crile, K. S. Dod et al., "Remissions in leukemia of childhood following acute infectious disease. Staphylococcus and streptococcus, varicella, and feline panleukopenias," Cancer, vol. 6, no. 3, pp. 591-605, 1953.

[2] S. J. Russell and K. W. Peng, "Measles virus for cancer therapy," Measles, vol. 330, pp. 213-241, 2009.

[3] D. Wodarz, "Computational modeling approaches to the dynamics of oncolytic viruses," WIREs Systems Biology and Medicine, vol. 8, no. 3, pp. 242-252, 2016.

[4] D. Wodarz and N. Komarova, "Towards predictive computational models of oncolytic virus therapy: basis for experimental validation and model selection," PLoS One, vol. 4, no. 1, Article ID e4271, 2009.

[5] N. L. Komarova and D. Wodarz, "ODE models for oncolytic virus dynamics," Journal of Theoretical Biology, vol. 263, no. 4, pp. 530-543, 2010.

[6] D. Wodarz, "Viruses as antitumor weapons: defining conditions for tumor remission," Cancer Research, vol. 61, pp. 3501-3507, 2001.

[7] A. Ashyani, H. Mohammadinejad, and O. Rabieimotlagh, "Hopf bifurcation analysis in a system for cancer virotherapy with effect of the immune system," Jordanian Journal of Mathematics and Statistics, vol. 9, pp. 93-115, 2016.

[8] Z. Bajzer, T. Carr, K. Josić, S. J. Russell, and D. Dingli, "Modeling of cancer virotherapy with recombinant measles viruses," Journal of Theoretical Biology, vol. 252, no. 1, pp. 109-122, 2008.

[9] J. P Tian, "The replicability of oncolytic virus: defining conditions in tumor virotherapy," Mathematical Biosciences and Engineering, vol. 8, pp. 841-60, 2011.

[10] M. I. Titze, J. Frank, M. Ehrhardt, S. Smola, N. Graf, and T. Lehr, "A generic viral dynamic model to systematically characterize the interaction between oncolytic virus kinetics and tumor growth," European Journal of Pharmaceutical Sciences, vol. 97, pp. 38-46, 2017.

[11] D. Ikawati, W. Kusumawinahyu, and T. Trisilowati, "A dynamical analysis on a tumour virotherapy model with standard incident rate," Journal of Tropical Life Science, vol. 7, no. 1, pp. 16-22, 2017.

[12] A. L. Jenner, A. C. Coster, P. S. Kim, and F. Frascoli, "Treating cancerous cells with viruses: insights from a minimal model for oncolytic virotherapy," Letters in Biomathematics, vol. 5, pp. s117-s136, 2018.

[13] T. A. Phan and J. P. Tian, "The role of the innate immune system in oncolytic virotherapy," Computational and Mathematical Methods in Medicine, vol. 2017, Article ID 6587258, 2017.

[14] L. R. Paiva, C. Binny, S. C. Ferreira, and M. L. Martins, "A multiscale mathematical model for oncolytic virotherapy," Cancer Research, vol. 69, no. 3, pp. 1205-1211, 2009.

[15] S. O. Freytag, K. R. Rogulski, D. L. Paielli, J. D. Gilbert, and J. H. Kim, "A novel three-pronged approach to kill cancer cells selectively: concomitant viral, double suicide gene, and radiotherapy," Human Gene Therapy, vol. 9, no. 9, pp. 1323-1333, 1998. 
[16] Y. Touchefeu, G. Vassaux, and K. J. Harrington, "Oncolytic viruses in radiation oncology," Radiotherapy and Oncology, vol. 99, no. 3, pp. 262-270, 2011.

[17] L. You, C. T. Yang, and D. M. Jablons, "ONYX-015 works synergistically with chemotherapy in lung cancer cell lines and primary cultures freshly made from lung cancer patients," Cancer Research, vol. 60, pp. 1009-1013, 2000.

[18] D. Dingli, M. D. Cascino, K. Josić, S. J. Russell, and Z. Bajzer, "Mathematical modeling of cancer radiovirotherapy," Mathematical Biosciences, vol. 199, no. 1, pp. 55-78, 2006.

[19] Y. Tao and Q. Guo, "A free boundary problem modelling cancer radiovirotherapy," Mathematical Models and Methods in Applied Sciences, vol. 17, no. 8, pp. 1241-1259, 2007.

[20] W. Waseem, M. Sulaiman, A. Alhindi, and H. Alhakami, "A soft computing approach based on fractional order DPSO algorithm designed to solve the corneal model for eye surgery," IEEE Access, vol. 8, Article ID 61576, 2020.

[21] A. Khan, M. Sulaiman, H. Alhakami, and A. Alhindi, "Analysis of oscillatory behavior of heart by using a novel neuroevolutionary approach," IEEE Access, vol. 8, Article ID 86674, 2020.

[22] A. Ahmad, M. Sulaiman, A. Alhindi, and A. J. Aljohani, "Analysis of temperature profiles in longitudinal fin designs by a novel neuroevolutionary approach," IEEE Access, vol. 8, Article ID 113285, 2020.

[23] M. F. Khan, M. Sulaiman, C. A. Tavera Romero, and A. Alkhathlan, "A hybrid metaheuristic based on neurocomputing for analysis of unipolar electrohydrodynamic pump flow," Entropy, vol. 23, no. 11, p. 1513, 2021.

[24] I. Jadoon, M. A. Z. Raja, M. Junaid, A. Ahmed, A. u. Rehman, and M. Shoaib, "Design of evolutionary optimized finite difference based numerical computing for dust density model of nonlinear Van-der Pol Mathieu's oscillatory systems," Mathematics and Computers in Simulation, vol. 181, pp. 444-470, 2021.

[25] M. F. Khan, M. Sulaiman, C. A. Tavera Romero, and A. Alkhathlan, "Falkner-Skan flow with stream-wise pressure gradient and transfer of mass over a dynamic wall," Entropy, vol. 23, no. 11, p. 1448, 2021.

[26] Z. Sabir, M. A. Z. Raja, M. R. Ali, A. Arbi, and M. Kristiawan, "An efficient computational numerical approach for nonlinear mathematical influenza disease modelling," Research Square, vol. 2021, pp. 2-34, 2021.

[27] M. Shoaib, M. A. Z. Raja, M. T. Sabir et al., "A stochastic numerical analysis based on hybrid NAR-RBFs networks nonlinear SITR model for novel COVID-19 dynamics," Computer Methods and Programs in Biomedicine, vol. 202, Article ID 105973, 2021.

[28] M. Umar, Z. Sabir, M. A. Z. Raja, and Y. G. Sánchez, "A stochastic numerical computing heuristic of SIR nonlinear model based on dengue fever," Results in Physics, vol. 19, Article ID 103585, 2020.

[29] A. H. Bukhari, M. Sulaiman, M. A. Z. Raja, S. Islam, M. Shoaib, and P. Kumam, "Design of a hybrid NAR-RBFs neural network for nonlinear dusty plasma system," Alexandria Engineering Journal, vol. 59, no. 5, pp. 3325-3345, 2020.

[30] A. Miller and S. J. Russell, "The use of the NIS reporter gene for optimizing oncolytic virotherapy," Expert Opinion on Biological Therapy, vol. 16, no. 1, pp. 15-32, 2016.

[31] M. M. Samy, M. I. Mosaad, and S. Barakat, "Optimal economic study of hybrid PV-wind-fuel cell system integrated to unreliable electric utility using hybrid search optimization technique," International Journal of Hydrogen Energy, vol. 46, no. 20, pp. 11217-11231, 2021.

[32] I. Ahmed, G. U. Modu, A. Yusuf, P. Kumam, and I. Yusuf, "A mathematical model of Coronavirus Disease (COVID-19) containing asymptomatic and symptomatic classes," Results in Physics, vol. 21, Article ID 103776, 2021.

[33] M. Zomorodi-moghadam, M. Abdar, Z. Davarzani, X. Zhou, P. Pławiak, and U. R. Acharya, "Hybrid particle swarm optimization for rule discovery in the diagnosis of coronary artery disease," Expert Systems, vol. 38, Article ID e12485, 2021.

[34] H. Alrabaiah, M. Arfan, K. Shah, I. Mahariq, and A. Ullah, "A comparative study of spreading of novel corona virus disease by using fractional order modified SEIR model," Alexandria Engineering Journal, vol. 60, no. 1, pp. 573-585, 2021.

[35] A. S. V. Vasconcelos, L. S. B. Silva, R. T. N. Cardoso, and J. L. Acebal, "Optimization of a rainfall dependent model for the seasonal Aedes aegypti integrated control: a case of Lavras/ Brazil," Applied Mathematical Modelling, vol. 90, pp. 413-431, 2021.

[36] G. P. Karev, A. S. Novozhilov, and E. V. Koonin, "Mathematical modeling of tumor therapy with oncolytic viruses: effects of parametric heterogeneity on cell dynamics," Biology Direct, vol. 1, pp. 30-19, 2006.

[37] S. M. Al-Tuwairqi, N. O. Al-Johani, and E. A. Simbawa, "Modeling dynamics of cancer radiovirotherapy," Journal of Theoretical Biology, vol. 506, Article ID 110405, 2020.

[38] J.-H. Huang, S.-N. Zhang, K.-J. Choi et al., "Therapeutic and tumor-specific immunity induced by combination of dendritic cells and oncolytic adenovirus expressing IL-12 and 41BBL," Molecular Therapy, vol. 18, no. 2, pp. 264-274, 2010.

[39] L. Edelstein-Keshet, Mathematical Models in Biology, SIAM, Philadelphia, PA, USA, 2005.

[40] M. W. Hirsch, S. Smale, and R. L. Devaney, Differential Equations, Dynamical Systems, and an Introduction to Chaos, Academic Press, Cambridge, MA, USA, 2012.

[41] A. Friedman, J. P. Tian, G. Fulci, E. A. Chiocca, and J. Wang, "Glioma virotherapy: effects of innate immune suppression and increased viral replication capacity," Cancer Research, vol. 66, no. 4, pp. 2314-2319, 2006.

[42] S. Mirjalili, "SCA: a Sine Cosine algorithm for solving optimization problems," Knowledge-Based Systems, vol. 96, pp. 120-133, 2016. 